\title{
Instability regimes in the primary breakup region of planar coflowing sheets
}

\author{
D. Fuster ${ }^{1,} \dagger$, J.-P. Matas ${ }^{2}$, S. Marty ${ }^{2}$, S. Popinet ${ }^{3}$, J. Hoepffner ${ }^{1}$, \\ A. Cartellier ${ }^{4}$ and S. Zaleski ${ }^{1}$ \\ ${ }^{1}$ CNRS (UMR 7190), Université Pierre et Marie Curie, Institut Jean le Rond d'Alembert, \\ 75005, Paris, France \\ ${ }^{2}$ Université de Grenoble Alpes, LEGI, F-38000 Grenoble, France \\ ${ }^{3}$ NIWA National Institute of Water and Atmospheric Research, PO Box 14-901 Kilbirnie, Wellington, \\ New Zealand \\ ${ }^{4}$ CNRS, LEGI, F-38000 Grenoble, France
}

(Received 26 February 2013; revised 23 September 2013; accepted 4 October 2013)

This article investigates the appearance of instabilities in two planar coflowing fluid sheets with different densities and viscosities via experiments, numerical simulation and linear stability analysis. At low dynamic pressure ratios a convective instability is shown to appear for which the frequency of the waves in the primary atomization region is influenced by both liquid and gas velocities. For large dynamic pressure ratios an asymptotic regime is obtained in which frequency is solely controlled by gas velocity and the instability becomes absolute. The transition from convective to absolute is shown to be influenced by the velocity defect induced by the presence of the separator plate. We show that in this regime the splitter plate thickness can also affect the nature of the instability if it is larger than the gas vorticity thickness. Computational and experimental results are in agreement with the predictions of a spatio-temporal stability analysis.

Key words: absolute/convective instability, aerosols/atomization, gas/liquid flows

\section{Introduction}

The mechanisms leading to the breakup in gas-assisted atomization are still poorly understood. A typical situation where this configuration can be found is that of coaxial atomizers (Lasheras, Villermaux \& Hopfinger 1998; Lasheras \& Hopfinger 2000): a mixing layer is created when a fast gas jet and a slower liquid jet meet downstream of a splitter plate. The wavy structure appearing downstream of the separator plate has been thoroughly investigated experimentally, e.g. Marmottant \& Villermaux (2004) and Ben Rayana, Cartellier \& Hopfinger (2006), and is generally believed to arise from a Kelvin-Helmholtz instability of the mixing layer, but controlled by the thickness of the gas vorticity layer $\delta_{g}$ (Rayleigh 1900; Raynal 1997; Marmottant \& Villermaux 2004; Eggers \& Villermaux 2008).

The characteristics of the waves appearing in the primary atomization region have been traditionally discussed in terms of the linear stability of a base flow (see for 


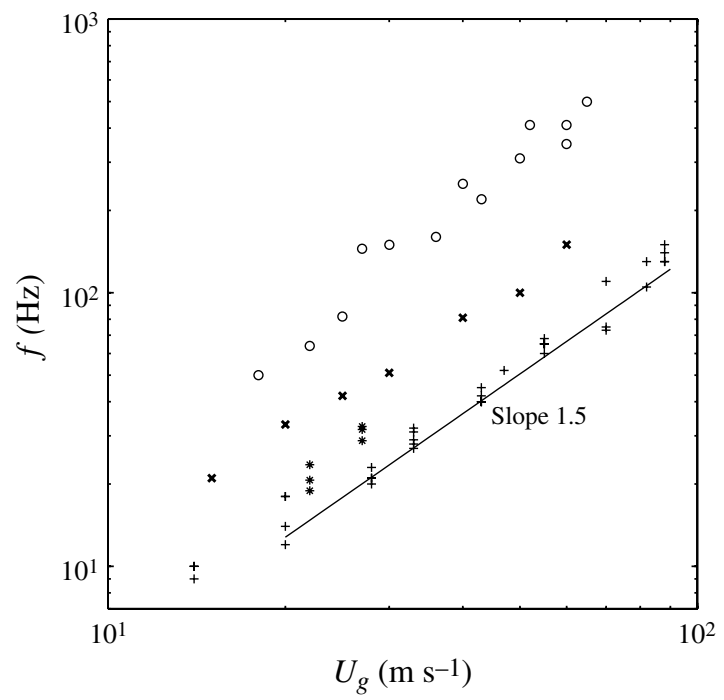

FIGURE 1. Experimental frequency versus gas velocity for various sets of experiments with $M>4$. Data obtained by: + , Raynal (1997) $M=[4.2: 2115]$; o, Marmottant \& Villermaux (2004) $M=[4: 108]$; $\times$, Ben Rayana (2007) $M=16$; and $*$, Matas, Marty \& Cartellier (2011) $M=\rho_{g} U_{g}^{2} / \rho_{l} U_{l}^{2}=[5: 14]$. The most unstable frequency is shown to scale as $U_{g}^{3 / 2}$ for large $M$.

instance Gordillo, Perez-Saborid \& Gañan-Calvo 2001). Based on this approach, several authors have tried to obtain accurate predictions of the wavelength, wave velocity and growth rate. In particular, inviscid linear stability analysis predicts a wavelength scaling as $\lambda \sim \sqrt{\left(\rho_{l} / \rho_{g}\right)} \delta_{g}$, and a growth rate scaling as $\omega_{i} \sim$ $\left(\rho_{g} / \rho_{l}\right)\left(U_{g} / \delta_{g}\right)$, where $\rho_{g}$ and $\rho_{l}$ are respectively the gas and liquid densities and $U_{g}$ the gas velocity (Raynal 1997; Marmottant \& Villermaux 2004). Frequency can be derived from this wavelength via the Dimotakis velocity $U_{D}=\left(\sqrt{\rho_{l}} U_{l}+\sqrt{\rho_{g}} U_{g}\right) /$ $\left(\sqrt{\rho_{g}}+\sqrt{\rho_{l}}\right)$ (Dimotakis 1986), which corresponds to the velocity of a frame in which liquid and gas dynamic pressures are balanced: $f \sim U_{D} / \lambda$. In the limit of large dynamic pressure ratios $M$ this scaling becomes $f \sim \sqrt{\left(\rho_{g} / \rho_{l}\right)} U_{g} / \lambda \sim \rho_{g} / \rho_{l} U_{g} / \delta_{g} \sim$ $U_{g}^{3 / 2}$. Figure 1 shows that the experimental frequencies of Raynal (1997), Marmottant \& Villermaux (2004), Ben Rayana (2007) and Matas et al. (2011) follow this scaling law relatively well. Note that only data for $M>4$ are shown: for smaller $M$ liquid velocity has a strong influence on frequency.

Although experimental wavelengths or frequencies follow this scaling law when gas and liquid velocities are varied, there is a discrepancy in the predicted values: experimental frequencies are larger than values predicted by inviscid analysis (Marmottant \& Villermaux 2004; Ben Rayana et al. 2006; Ben Rayana 2007) by a factor 2 or 3 . Based on the observation that the splitter plate has a strong impact on the initial velocity profile, Matas et al. (2011) extended this inviscid analysis to velocity profiles with a zero interface velocity: they obtain a reasonable agreement with experimental frequencies measured in a planar mixing layer set-up for low dynamic pressure ratios, but their model strongly underestimates the experimental spatial growth rate measured just downstream of the splitter plate (Matas et al. 2011). Yecko, Zaleski \& Fullana (2002) and Boeck \& Zaleski (2005) studied the stability 
of the two-phase mixing layer via temporal linear stability calculations based on the Orr-Sommerfeld equations: the most unstable mode predicted by this approach, which is viscous, has a growth rate closer to experiments, but its frequency is then too large. Transient growth theory has been used by Yecko \& Zaleski (2005). The main result of this investigation is to show that in some cases three-dimensional perturbations are more unstable than two-dimensional ones. However, a systematic comparison of longitudinal wavelength with experimental results has not been performed in that work so the transient growth effects have not been compared to the experimental results.

While the previous works were either temporal or spatial linear stability studies, some authors have more recently investigated the temporal-spatial problem for multiphase flows. For instance, Juniper \& Candel (2003), Juniper (2006), Rees \& Juniper (2010) and Juniper, Tammisola \& Lundell (2011) have investigated the effect of confinement on the stability of two-dimensional shear inviscid flows. Other flow configurations have been considered by Sahu et al. (2007), Ó'Náraigh, Spelt \& Zaki (2011) and Ó'Náraigh, Spelt \& Shaw (2013). In the context of atomization, Otto, Rossi \& Boeck (2013) developed the spatio-temporal theory to clarify the nature of the instability (i.e. determine whether the instability is convective or if it is a global mode) and the influence of the base flow profile on the stability results, namely the influence of a velocity defect such as proposed by Matas et al. (2011). They compared their predictions for frequency and spatial growth rate of the instability to the experimental data of Marmottant \& Villermaux (2004) and Matas et al. (2011). They found a relatively good agreement on frequencies for several sets of conditions, though this agreement is not systematic. The agreement with the growth rate is less satisfactory, but still better than that achieved using inviscid studies. An important result from their study is the relevance of the convective/global instability transition for the conditions of experiments: they find that experimental data span both regimes, and therefore emphasize the need for a spatio-temporal analysis. It is important to note that, unlike in Juniper's works (Juniper \& Candel 2003; Juniper 2006; Rees \& Juniper 2010; Juniper et al. 2011), the identification of an absolute instability is not attributed to the confinement of the system, but to the presence of a velocity defect in the base flow profile induced by the splitter plate.

Few studies compare numerical predictions with either experimental or theoretical data. Only in recent years do we start finding in the literature detailed simulations of atomization process (Bianchi et al. 2005; Menard, Tanguy \& Berlemont 2007; Gorokhovski \& Herrmann 2008; Shinjo \& Umemura 2010, 2011). Unfortunately, numerical simulations are exceedingly expensive for conditions relevant to experiments in air/water conditions. In the context of two planar coflowing fluid sheets, previous numerical results point to a convective instability (Fuster et al. 2009b) while a different instability mechanism, with large, presumably nonlinear perturbations is reported by Fuster et al. (2009a) and Agbaglah et al. (2011).

The present work is aimed at gaining new insight into the underlying mechanisms controlling the appearance of instabilities in the primary atomization region. To this end, we use a numerical tool to investigate in detail a wide range of conditions as a function of the dynamic pressure ratio and gas velocity. The results obtained from the numerical analysis are discussed in terms of absolute versus convective instability, comparing the conclusions obtained to new measurements included in this manuscript as well as to theoretical predictions. We pay special attention to the influence of the splitter plate on the nature of the instability as well as the spectra obtained from numerical simulations and experiments. The paper is structured as follows. In $\S 2$ 


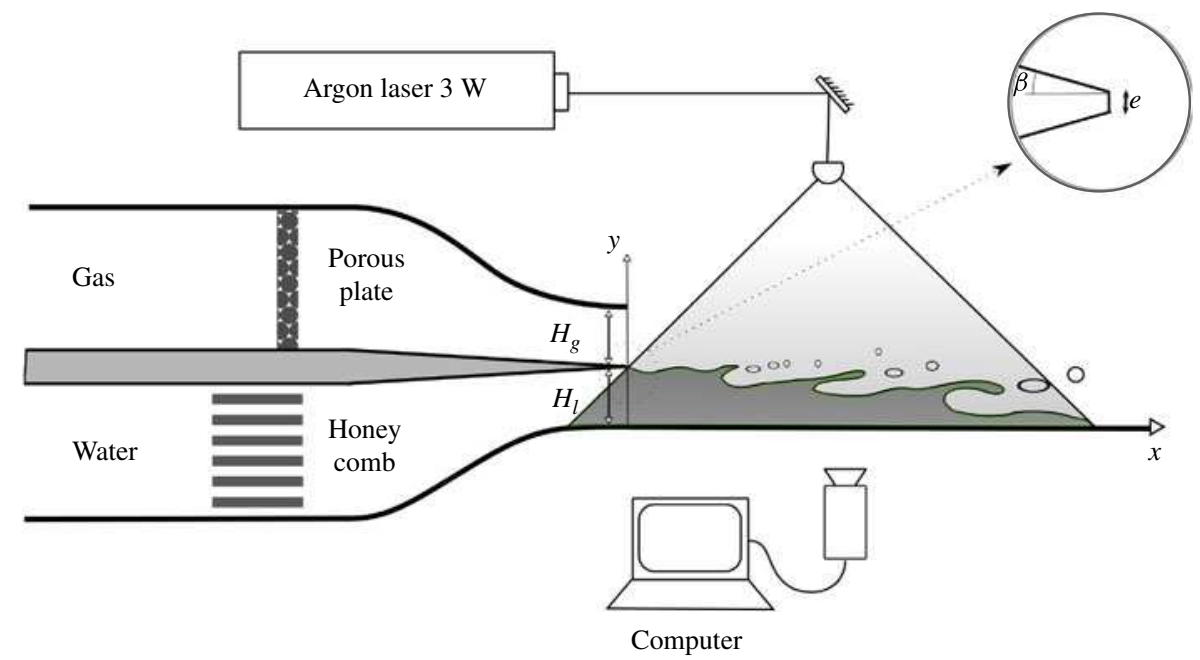

FIGURE 2. (Colour online) Sketch of the experimental set-up. Two splitter plates are used in the experiment: $e=0.2 \mathrm{~mm}$ and $\beta=4.15^{\circ}$, and $e=2.2 \mathrm{~mm}$ and $\beta=4.1^{\circ}$.

we describe the experimental set-up to introduce the physical problem. In $\S 3$ we briefly describe the currently available spatio-temporal linear theory introduced by Otto et al. (2013) for the theoretical predictions of the observed frequencies. In $\S 4$ we give details about the numerical tool used in this work for the simulation of primary atomization. The results are presented in $\$ \S 5$ and 6 of the paper: in the first we discuss the atomization regimes found for moderate density ratios, where it is possible to perform a detailed analysis of the atomization process. Second, the results are extended to air/water conditions where the new measurements serve to validate some of the conclusions obtained from the numerical analysis.

\section{Experimental set-up and measurement techniques}

The experimental set-up is a mixing layer experiment: a water stream is destabilized by a parallel fast air stream. The height of the air exit channel, $H_{g}$, is $1 \mathrm{~cm}$ and the liquid height, $H_{l}$, can be varied between 0.5 and $10.5 \mathrm{~mm}$ (the bottom plate can be moved). In the present study the height is kept at $H_{l}=6.4 \mathrm{~mm}$, giving a contraction ratio of 8 in the liquid channel. The contraction ratio in the gas channel is 10 . We use two splitter plates of $e=0.2 \mathrm{~mm}$ and $\beta=4.15^{\circ}$, and $e=2.2 \mathrm{~mm}$ and $\beta=4.1^{\circ}$, where $e$ is splitter plate thickness and $\beta$ its slope (or opening half-angle). In this later case the position of the bottom plate is adjusted in order to maintain a constant $H_{l}=6.4 \mathrm{~mm}$. The channel width is $10 \mathrm{~cm}$. The liquid flow rate is maintained via an overflowing reservoir. The air stream is generated from a blowing machine, and regulated in temperature with a heat exchanger so that it is delivered at ambient temperature and pressure conditions. Both streams issue from symmetrical convergent channels. We use honeycombs on the liquid side, and a porous plate on the gas side in order to damp velocity fluctuations (see figure 2).

Stability analyses have identified the vorticity thickness $\delta_{g}=\Delta U /\left.(\mathrm{d} U / \mathrm{d} y)\right|_{\max }$ as a crucial parameter. Hence we took care to measure it as precisely as possible as a function of gas velocity, with liquid flow turned off for obvious practical reasons. Hotwire measurements established that the vorticity thickness varies as $\delta_{g} / H_{g}=5.2 / \sqrt{R e}$, 


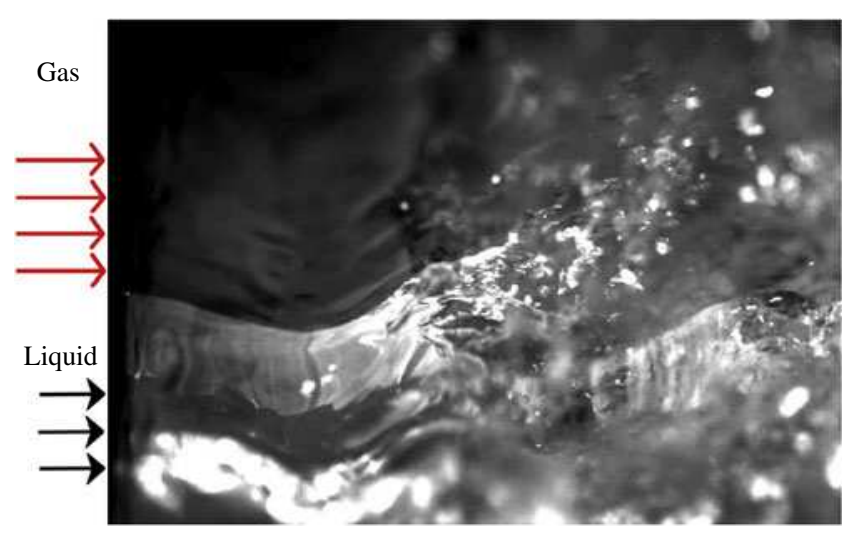

FIGURE 3. (Colour online) Visualization of the shear instability with both a laser slice and white light: $U_{g}=25 \mathrm{~m} \mathrm{~s}^{-1}$ and $U_{l}=0.55 \mathrm{~m} \mathrm{~s}^{-1} ; e=0.2 \mathrm{~mm}$.

where the gas Reynolds number is based on the stream thickness $H_{g}: R e=H_{g} U_{g} / v_{\text {air }}$. Gas velocity is measured with a Pitot tube (diameter $6 \mathrm{~mm}$ ), positioned midway from the sidewalls and at mid-height of the gas stream. This measurement method was cross-checked with measurements both with a smaller Pitot tube (diameter $3 \mathrm{~mm}$ ) and with an air velocity transducer (TSI 8455). A McMillan mass flowmeter provides an additional measurement, with less accuracy, which is only used to check consistency of the Pitot measurement (note that the Pitot tube could be affected by droplets). Liquid flow rate, from which liquid velocity is deduced, is measured with a rotameter.

A longitudinal section of the interface is obtained using an argon laser. Thanks to fluorescein added in the liquid phase, the laser enhances the longitudinal variations of the interface, while white lighting clarifies the contours of the longitudinal section: it must be noted that the alignment of the wavefront confirms the two-dimensional symmetry of the instability for these flow conditions (figure 3 shows what can be seen when the laser slice is superposed with white light).

The visualization of the laser slice with a fast camera allows us to capture the position of the liquid/gas interface as a function of time. Figures 4 and 5 show typical interface profiles obtained with the technique described above. Even in the presence of drops (figure 5), the global evolution of the wave contour is still captured.

We perform measurements of the temporal oscillations of the interface position at different distances downstream from the separator plate. As previously observed by Marmottant \& Villermaux (2004) the velocity of the waves increases when distance from the nozzle grows. In Marmottant \& Villermaux (2004) the liquid is accelerated by gravity, whereas in the present experiment it is not. Nonetheless gas flow may induce an acceleration as well: because the amplitude of the waves is large compared to the gas vorticity thickness, gas flow induces a strong drag on waves formed by the instability once the amplitude of these waves has increased significantly. At the same time, growing waves impact on the gas flow, and the gas boundary thickness is seen to vary strongly spatially in particle image velocimetry (PIV) measurements (see for example figure 22 of Matas et al. 2011). Wave phase speed is therefore difficult to define unambiguously. Thus, we chose to focus on frequency measurements, which can be measured locally. Wavelength measurements would imply a measurement that stretches across a strongly varying gas/liquid flow. In order to determine if frequency varies spatially, spectra are computed at different downstream distances for a given 
(a)

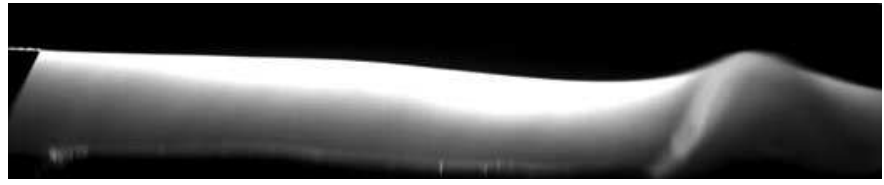

(b)

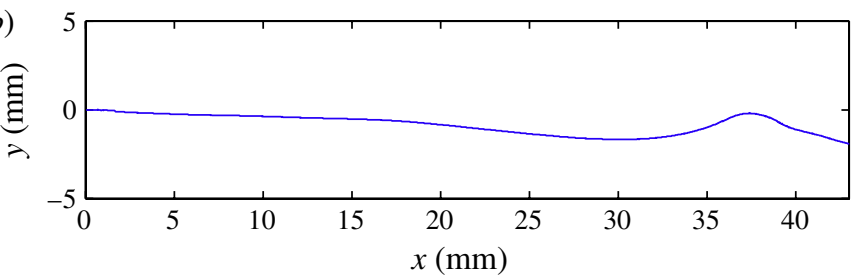

FIGURE 4. (Colour online) (a) Laser slice of the flow seeded with fluorescein. The white line on the left is a reflection on the upper surface of the splitter plate. (b) Interface extracted from the image: $U_{g}=10 \mathrm{~m} \mathrm{~s}^{-1}$ and $U_{l}=0.35 \mathrm{~m} \mathrm{~s}^{-1} ; e=0.2 \mathrm{~mm}$.

(a)

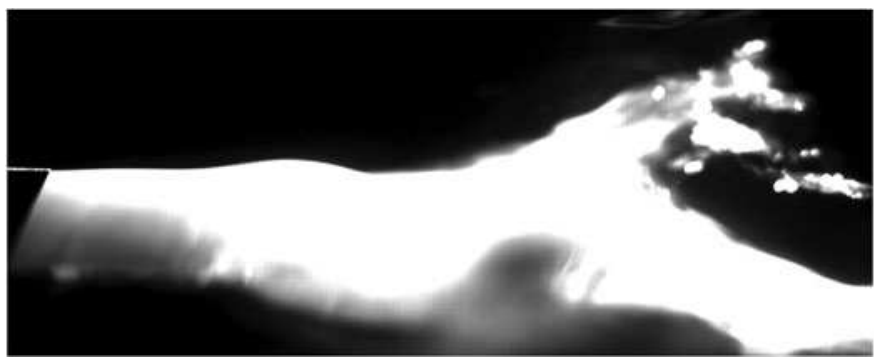

(b)

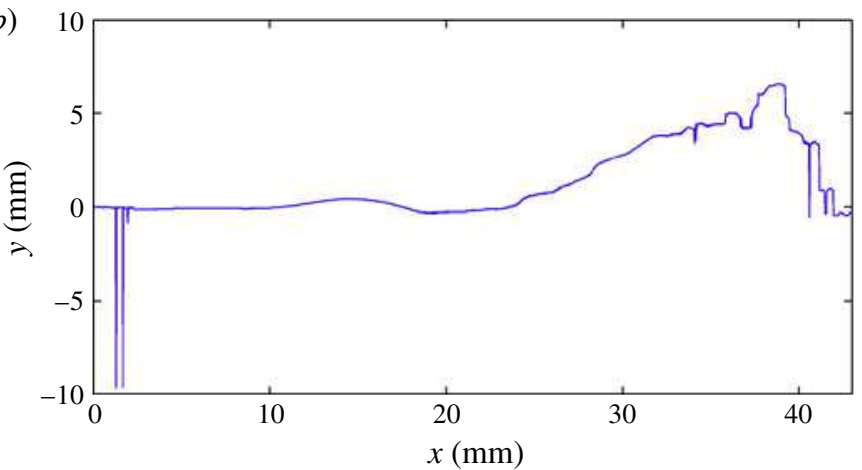

FIGURE 5. (Colour online) (a) Laser slice of the flow seeded with fluorescein. $(b)$ Interface location computed from the image: $U_{g}=20 \mathrm{~m} \mathrm{~s}^{-1}$ and $U_{l}=0.70 \mathrm{~m} \mathrm{~s}^{-1} ; e=0.2 \mathrm{~mm}$.

set of conditions. The frequency measurements are taken by applying a fast Fourier transform (FFT) algorithm to the time evolution of the interface height at a given downstream position. Figure 6 shows a typical experimental FFT measurement as a function of the downstream distance for a moderate $M=3.7$. Frequency does not vary with downstream position, and a single value can therefore be extracted for given gas/liquid velocities. 

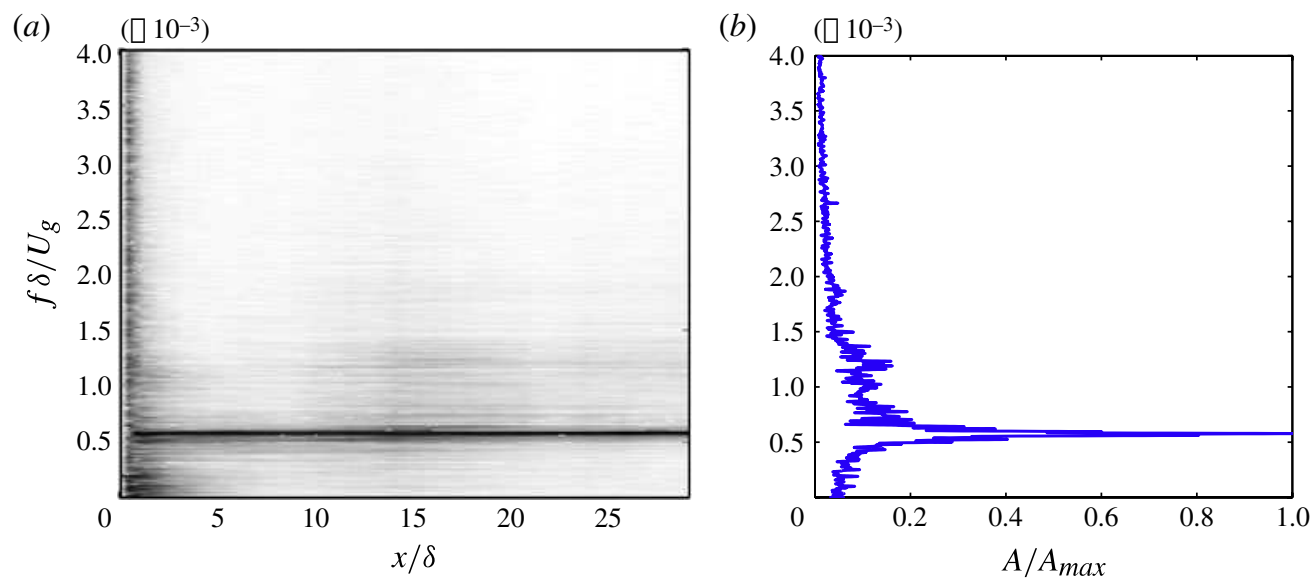

Figure 6. (Colour online) (a) Experimental spectrum as a function of the downstream position for $U_{g}=20 \mathrm{~m} \mathrm{~s}^{-1}$ and $M=3.7$ and splitter plate thickness $e=2.2 \mathrm{~mm}$. The maximum frequency remains unchanged with the distance. (b) Spectrum at $x / \delta_{g}=20$.

\section{Linear theory}

In this work we use the temporal and spatio-temporal results obtained using the analysis presented by Otto et al. (2013). For clarity, we briefly summarize the essence of the two theoretical approaches used as reference for the numerical and experimental results contained in this work.

\subsection{Temporal linear theory}

The temporal analysis solves for the temporal evolution of a perturbation with a given wavelength. This method is relatively simple to code and the results are also simpler to interpret than in the case of the spatio-temporal problem. Thus, for practical reasons, there is an interest in investigating the capability of temporal analysis to reproduce a real situation.

In the temporal problem, the streamlines are supposed to obey the following form:

$$
\psi_{j}(x, y, t)=\phi_{i}(y) \exp [\mathrm{i}(\alpha x-\omega t)],
$$

where $\alpha$ is the real wavenumber and $\omega$ is the complex frequency whose imaginary part represents the growth rate of a disturbance with a given $\alpha$. Accounting for viscosity, and using the gas boundary layer and the mean velocity $\left(U_{\text {avg }}=\left(U_{l}+U_{g}\right) / 2\right)$ to obtain non-dimensional quantities denoted with a subscript asterisk, the following non-dimensional velocity profile is used:

$$
\begin{gathered}
u_{l}^{*}(y)=-\left(1-\Delta U^{*}\right) \operatorname{erf}\left(\frac{y^{*}}{\delta_{l}^{*}}\right)+U_{0}^{*}\left[1+\operatorname{erf}\left(\frac{y^{*}}{\delta_{d}^{*}}\right)\right], \quad y^{*}<0, \\
u_{g}^{*}(y)=\left(1+\Delta U^{*}\right) \operatorname{erf}\left(y^{*}\right)+U_{0}^{*}\left[1-\operatorname{erf}\left(\frac{y^{*}}{\delta_{d}^{*}}\right)\right], \quad y^{*}>0,
\end{gathered}
$$

where $\delta_{l}^{*}$ represents the non-dimensional liquid boundary layer thickness, $\delta_{d}^{*}$ is the non-dimensional velocity defect thickness, $\Delta U^{*}$ is defined as

$$
\Delta U^{*}=\frac{U_{g}-U_{l}}{U_{g}+U_{l}}
$$




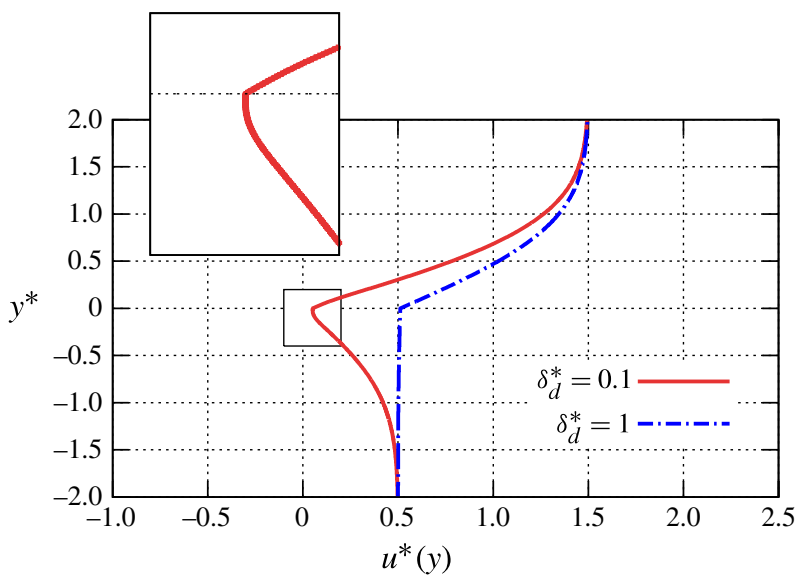

FIGURE 7. (Colour online) Example of the base flow profile used for the temporal and spatio-temporal calculations for $\Delta U^{*}=0.5, m=0.012$ and $\delta_{l}^{*}=1$.

$U_{g}$ and $U_{l}$ are the gas and liquid velocities far from the boundary layer, and $U_{0}^{*}$ can be obtained by applying continuity of shear stresses across the interface (Otto et al. 2013):

$$
U_{0}^{*}=\frac{m \delta_{l}^{*}\left(1+\Delta U^{*}\right)+\left(1-\Delta U^{*}\right)}{m+1} \delta_{d}^{*},
$$

where $m$ denotes the viscosity ratio between the two phases.

The parameter $\delta_{d}^{*}$ can be used as an adjustable parameter to mimic velocity profiles obtained from experimental and simulation results just behind the splitter plate. Note that this parameter will be changed in the analysis presented in order to evaluate the influence of the splitter-plate wake flow on the theoretical predictions. Figure 7 depicts the base flow profile for two different values of $\delta_{d}^{*}$. The limit $\delta_{d}^{*}=1$ represents the case of a splitter plate where the slip condition applies at the wall. When $\delta_{d}^{*}<1$, the velocity profile of (3.2) and (3.3) exhibits a minimum in the liquid phase (Otto et al. 2013), which allows inclusion of the velocity defect in the averaged profile while still keeping the continuity of shear stress at the interface. Note that for the simulations included in this work, the liquid boundary layer thickness is chosen to be equal to the gas boundary layer $\left(\delta_{l}^{*}=1\right)$.

\subsection{Spatio-temporal linear theory}

The analysis above is extended to the spatio-temporal problem, where the wavenumber is a complex quantity whose complex part represents the spatial growth rate at a given complex frequency $\omega$. When the flow is convectively unstable, the maximum spatial growth rate corresponds to the case where $\omega_{i}=0$. When the flow is absolutely unstable, spatial branches appear in the stability diagram that approach each other when $\omega_{i}$ is increased. After a critical $\omega_{i, c}$ the branches diverge. The critical point at which the branches touch is usually called the saddle point and provides the most unstable frequency that is expected to be observed in a real experiment. For further details about spatio-temporal linear analysis the reader is referred to the seminal works of Huerre \& Monkewitz (1985, 1990). Details about the application of the theoretical approach used here can be found in Otto et al. (2013). One important remark is that 
spatio-temporal theory results provide information about the nature of the instability and therefore it predicts the appearance of two different regimes depending on whether the instability is convective or absolute. Both temporal and spatio-temporal results will be included in this work as a reference for numerical and experimental results.

\section{Numerical tool}

For the numerical computations included in this work we use the Gerris flow solver, which has been shown to be an accurate solver for two-phase flows (Popinet 2003, 2009) and in particular, for capturing Kelvin-Helmholtz-like instabilities (Bagué et al. 2010).

This solver presents several appealing features that make it suitable for the simulation of atomization processes. It is based on a volume-of-fluid (VOF) technique, which handles naturally topological changes of the interface. It implements an accurate algorithm for the calculation of the surface tension force. Regarding the mesh, the code allows an automatic octree refinement based on user-defined criteria. In particular, for the simulations included in this work we adapt the grid according to the gradient of the VOF colour function and the norm of the vorticity. The first criterion allows us to control the degree of accuracy with which the interface is captured. The second one controls the level of resolution achieved to capture the turbulent vortices. Both criteria are also space-dependent in order to concentrate the computational effort in the zone near the injector.

Finally, the small-droplet to particle conversion algorithms described in Tomar et al. (2010) have also been used. The advantages are twofold: the mesh is significantly coarsened in regions where the jet has mainly broken into small fragments, and we avoid the introduction of large spurious numerical errors in the solution due to small liquid fragments that cannot be resolved with the mesh.

\section{Instability regime diagrams at moderate density ratios}

In this section, we set up a simplified scenario for numerical computations that allows us to gain new insight into the physical mechanisms leading to the appearance of waves in the primary atomization region. We show that some of the conclusions obtained from the analysis of this simplified scenario already capture some important experimental observations as well as theoretical predictions. In particular we focus on two important aspects: the transition from convective to absolute instability and the influence of the separator plate thickness on the observed frequencies.

\subsection{Problem set-up}

We perform various computations in a computational domain restricted to a relatively narrow region around and downstream of the splitter plate. The density ratio is chosen to be $r=\rho_{g} / \rho_{l}=0.1$. Using moderate density ratios and small domains allows much faster and longer simulations and therefore we can investigate the effect of several parameters, such as separator plate width and dynamic pressure ratio, based on reliable statistics obtained during relatively long times.

In order to perform long simulations, we restrict ourselves to two-dimensional computations. This is justified by experimental observations, where we clearly see that the flow remains almost two-dimensional in the region of interest (near the injector). Figure 8 contains an example of the computational domain used for the simulations included in this section as well as the boundary conditions. At the left side of the domain, Dirichlet boundary conditions are imposed for both gas and liquid velocities. 


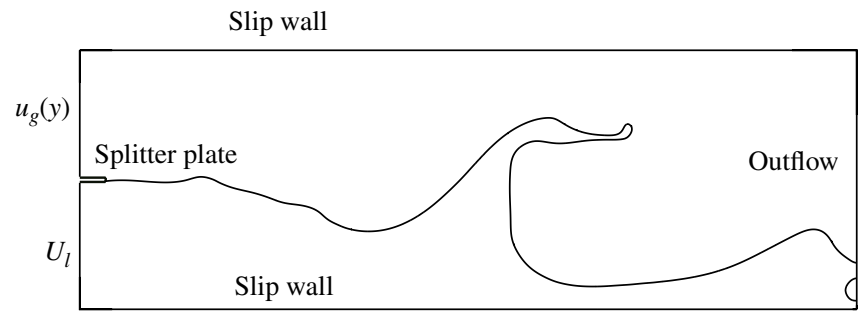

FIGURE 8. Computational domain and boundary conditions for the simulations at low density ratios $(r=0.1)$.

A splitter plate of thickness $e$ is placed between the two phases at the entrance where a non-slip boundary condition is used. The gas velocity profile at the entrance is:

$$
u_{g}(y)=\left(U_{g}+U_{g, \text { perturb }}\right) \operatorname{erf}\left(\frac{y+e / 2}{\delta_{g}}\right),
$$

where the system of coordinates is centred at the left of the domain and the middle of the separator plate, $U_{g, \infty}$ represents the velocity of the base flow and $U_{g, \text { perturb }}$ represents a perturbation on the gas velocity at the entrance. For reasons that become clear later, we introduce a disturbance on the velocity at a set of discrete frequencies, such that

$$
U_{g, \text { perturb }}=10^{-3} U_{g, \infty} \frac{\sum_{i=1}^{N} \sin \left(\mathrm{i} \Delta \omega_{i} t\right)}{N},
$$

where $\Delta \omega_{i} \delta_{g} / U_{g, \infty}=2 / 3$ determines the $N$ discrete forcing frequencies $(N=10)$. Because usually the thickness of the liquid boundary layer does not have an impact on the observed frequencies, the liquid velocity, $U_{l, \infty}$, is set to a constant value at the entrance. That is, we consider here the limiting case of $\delta_{l}=0$ at the inlet. Note that the only purpose is to remove $\delta_{l}$ from the list of parameters that has to be specified. A liquid boundary layer is always created just behind the injector as a consequence of the presence of the injector and the velocity difference between the gas and the liquid phase. Finally, the simulation domain size is $6 L \times 2 L$, with $L / e=30$. In this case, we adapt the mesh at the interface and also as a function of the vorticity and curvature. The minimum mesh size is set to $\Delta x / e=0.117$.

We start by discussing the influence of the dynamic pressure ratio on the nature of the observed instability. The dynamic pressure ratio is one of the most significant parameters in order to characterize atomization processes. Taking as reference the work of Ben Rayana (2007), we vary $M$ by changing $U_{l}$ at constant $U_{g}$. This is justified by the fact that the liquid boundary layer is not observed to play a role experimentally. Changing $U_{g}$ makes results more difficult to interpret given that the thickness of the gas boundary layer also changes in experiments.

We take as characteristic values of the process the gas velocity, the gas density and the thickness of the gas boundary layer. The non-dimensional values characterizing the system are contained in table 1 .

The simulations are run for a time equal to $t^{*}=t U_{g, \infty} / \delta_{g}=15000$ in order to obtain representative statistics of the dynamics of the system. The position of the interface is obtained at every time and at every position $x$, where $x$ represents the stream direction. 

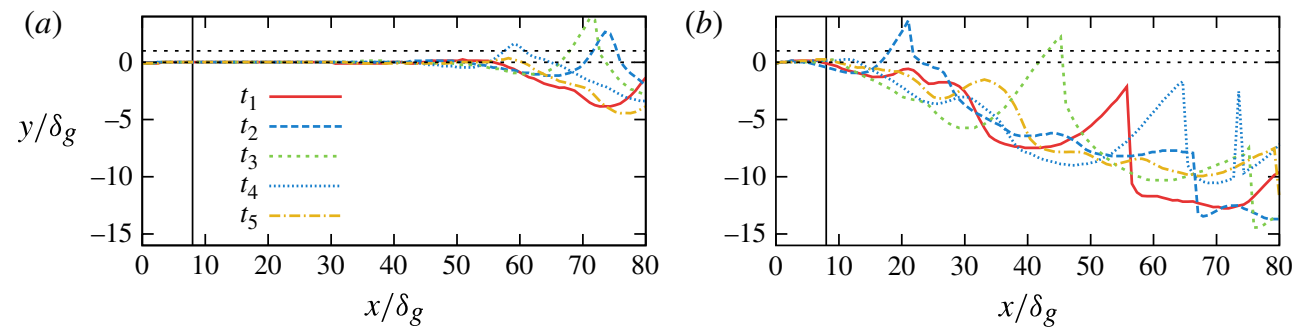

FIGURE 9. (Colour online) Interface position sampled at different times during one cycle $\left(\Delta t^{*}=3750\right)$. The axes have been stretched to facilitate the visualization. The vertical line represents the location where the interface is sampled to obtain the FFT. For reference we include as horizontal lines the thickness of the gas boundary layer at $x / \delta_{g}=0(a) M=1.25$; (b) $M=20$.

$$
\begin{array}{cccccc}
e / \delta_{g} & \mu_{l} / \mu_{g} & W e_{g} & R e_{g} & r & M \\
& & \frac{\rho_{g} U_{g}^{2} \delta_{g}}{\sigma} & \frac{\rho_{g} U_{g} \delta_{g}}{\mu_{g}} & \rho_{g} / \rho_{l} & \frac{\rho_{g} U_{g}^{2}}{\rho_{l} U_{l}^{2}} \\
& & 10 & 1000 & 0.1 & 1.25 \rightarrow 20
\end{array}
$$

TABLE 1. Simulation conditions for the analysis of atomization processes.

When the interface is located at different heights for a given $x$, the minimum value among them is chosen as a representative value. Choosing a distance from the injector, the temporal evolution at a given location is obtained and, finally, the FFT is applied to find the characteristic frequency of the interface oscillation at this point. Figure 9 depicts typical interface profiles along the downstream direction obtained for low (figure $9 a$ ) and large dynamic pressure ratios (figure 9b). The two different regimes observed are discussed in the next two subsections.

\subsection{Low dynamic pressure ratio regime: the $N A$ regime}

When the dynamic pressure ratio is small, the interface disturbances close to the splitter plate are very small (figure $9 a$ ). Thus linear theory results are expected to be applicable and, as expected for noise amplifier systems, all the forcing frequencies with a positive growth rate are clearly observed. The amplitude of the peaks depends on the growth rate, which ultimately is a function of the forcing frequency, e.g. Bagué et al. (2010). In order to compare to the theoretical results, we use the Dimotakis velocity

$$
U_{D}=\frac{U_{g} \sqrt{\rho_{g}}+U_{l} \sqrt{\rho_{l}}}{\sqrt{\rho_{g}}+\sqrt{\rho_{l}}}
$$

to obtain a measure of the non-dimensional wavenumber from $f$. In agreement with linear theory predictions, figure 10 reveals that the FFT of the interface position obtained from numerical simulations shows clear peaks at the forcing frequencies $\omega_{i}$. The analysis of the results obtained from the spatio-temporal linear problem predicts relatively well the range of frequencies that are most amplified. For reference, the 


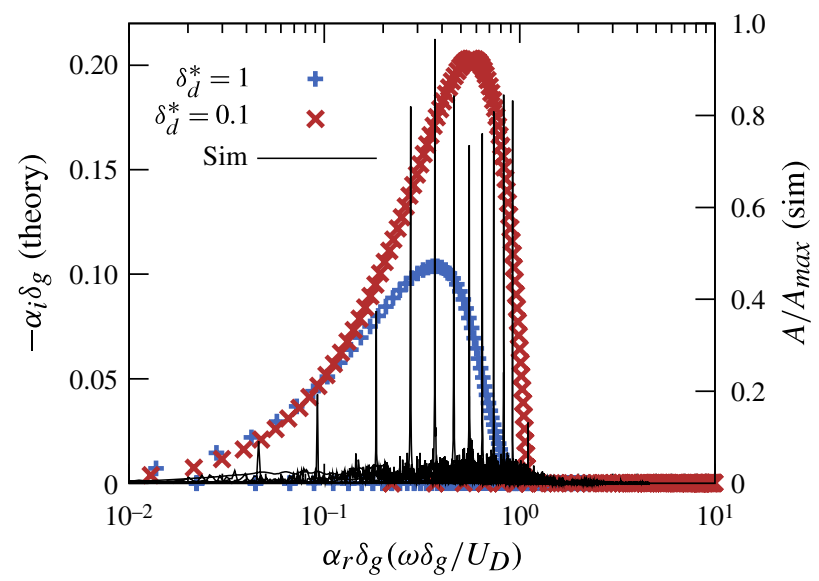

FIGURE 10. (Colour online) $M=1.25$. - , Fourier spectra of the temporal evolution of the interface at $x / e=8$. The frequency analysis also contains the linear theory results obtained from the spatio-temporal analysis for a velocity profile without defect $(+)$ and taking into account the effect of the velocity defect $(\times)$. The discrete forcing frequencies are clearly observed in the spectra at low dynamic pressure ratios. A convective instability is observed. The most unstable frequencies seem to be correctly predicted by linear theory analysis for this case.

(a)

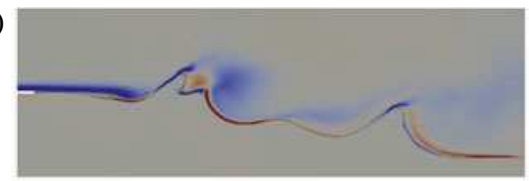

(c)

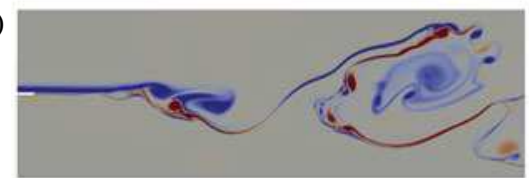

(b)

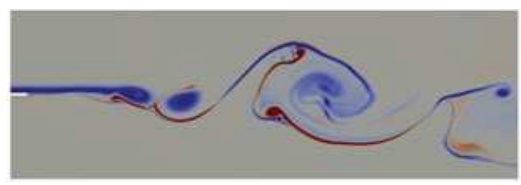

(d)

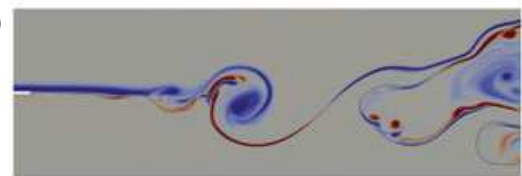

FIGURE 11. (Colour online) Interface position and vorticity field for $M=20$ during one cycle $\left(\Delta t^{*}=3750\right):(a) t_{0}^{*} ;(b) t_{0}^{*}+\Delta t^{*} ;(c) t_{0}^{*}+2 \Delta t^{*} ;(d) t_{0}^{*}+3 \Delta t^{*}$.

theoretical predictions obtained without velocity defect $\left(\delta_{d}=1\right)$ and with velocity defect $\left(\delta_{d}=0.1\right)$ are included. We conclude that for low dynamic pressure ratios, the linear theory results predict with a reasonable degree of accuracy the most unstable frequencies in the region where the amplitudes are small. We name this regime a noise amplifier (NA).

\subsection{Large dynamic pressure ratio regime: the $G$ regime}

Figure 11 depicts a typical sequence of the appearance and propagation of a large wave at large dynamic pressure ratios $(M=20)$. A large wave appears cyclically in the simulation domain and propagates downstream. In general, the wave grows significantly when its top goes beyond the gas boundary layer. When the wave is large enough, the suction induced by the wave growth influences the height of the interface upstream. This phenomena can be observed at $M=20$ for $10<x / \delta_{g}<20$ (figure 11). 

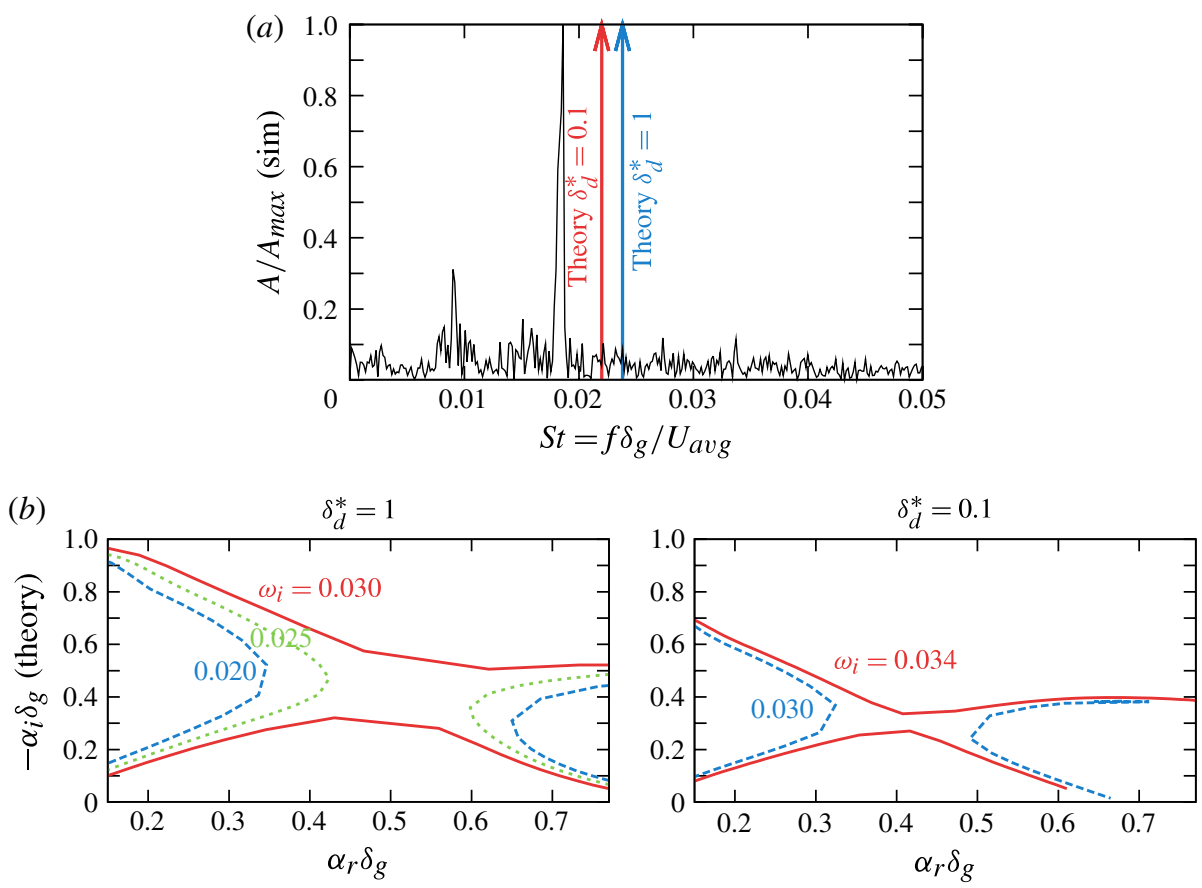

FIGURE 12. (Colour online) $M=20$. (a) Fourier spectra of the temporal evolution of the interface height at $x / e=8$ obtained from simulation. Arrows indicate the theoretical spatiotemporal predictions of the real frequency corresponding to the saddle point taking into account the velocity defect (left arrow) and neglecting it (right arrow). (b) $\alpha_{i}$ versus $\alpha_{r}$ diagram from the spatio-temporal analysis taking into account the velocity defect (right plot) and neglecting it (left plot). Simulation results show a clear peak frequency, which indicates the appearance of an absolute instability for large dynamic pressure ratios. This observation is consistent with the stability diagrams obtained from the analysis of the spatio-temporal problem.

As the wave propagates downstream, the liquid level recovers until it reaches the initial level and the next wave appears.

Figure 12 depicts the Fourier spectra of the temporal evolution of the interface at a distance from the separator plate equal to $x / e=8$ for the high dynamic pressure ratio. We emphasize that the forcing frequencies are the same as for the case of low dynamic pressure ratio. Remarkably the spectrum of the interface position evolution is significantly different. The forcing frequencies at the inlet are no longer visible. Instead, a clear peak in the spectrum appears which is identified with a resonant frequency. In agreement with the numerical results, the analysis of the spatio-temporal problem reveals the global nature of the instability. Thus, we can state that for this particular example the behaviour predicted theoretically is in agreement with the spectra found from numerical results. When comparing the values of the most unstable frequencies one can see that, despite the likely presence of nonlinear effects, one can get a first approximation of the expected frequencies using the linear theory results. However, linear theory results overestimate the wavenumbers obtained from computations. For the large dynamic pressure ratios tested in this work, including a velocity profile with a velocity defect that accounts for the presence of the separator plate seems to slightly improve the theoretical predictions, although these changes do 


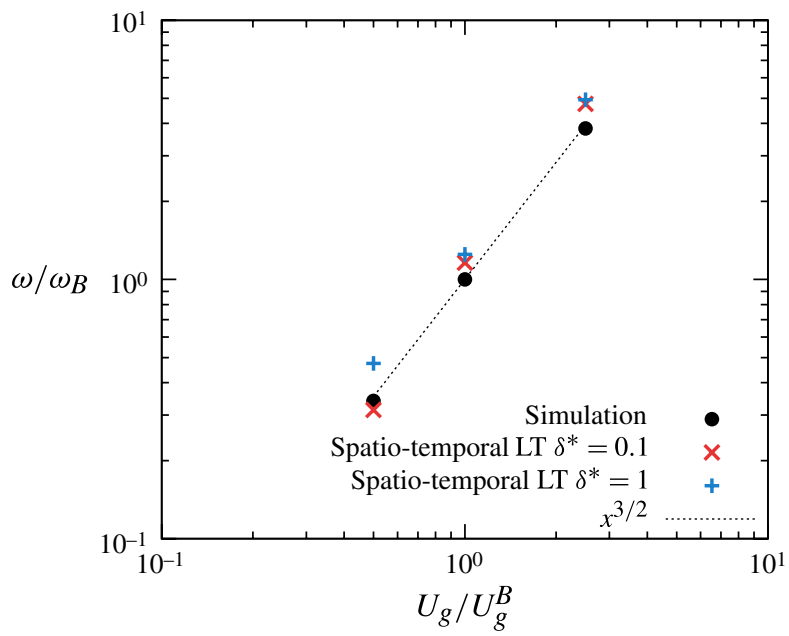

FIGURE 13. (Colour online) Peak frequency as a function of the gas velocity at large dynamic pressure ratios. $U_{g}^{B}$ represents the gas velocity previously used to perform the simulations at constant gas velocity (table 1 ) and $M=20 . \omega_{B}$ is the observed frequency at these conditions. LT denotes linear theory.

not seem important enough to explain the quantitative disagreement of theoretical and simulation results.

Another important parameter to consider is the scaling of the most unstable frequency as a function of the gas velocity obtained experimentally (figure 1). To gain insight into the gas velocity influence we perform simulations for three different gas velocities. The liquid velocity is modified to keep the dynamic pressure ratio constant and equal to 20. In addition, trying to mimic the observed experimental dependence of the frequency on the velocity of the gas, the thickness of the boundary layer is also modified according to the experimentally observed correlation, where $\delta_{g} \propto U_{g}^{-1 / 2}$. Figure 13 depicts the peak frequency from numerical computations versus the gas velocity. For reference we make both velocity and frequency non-dimensional with respect to the values obtained from the simulation at $M=20$ (the rest of the conditions are defined in table 1). As can be seen, the scaling obtained from numerical computations fits a 3/2 slope well. It is also remarkable that spatio-temporal linear theory predictions capture well the scaling and the quantitative values of the frequencies observed. This feature of the stability code is very remarkable because the temporal linear stability analysis for inviscid flow only predicts the correct slope but not the quantitative values, whereas the extension of this analysis to viscous flows slightly improves the quantitative values but fails to capture the correct slope (Ben Rayana 2007). As said above, in this regime the most unstable frequency does not depend significantly either on the liquid velocity or on the dynamic pressure ratio. Thus, as the gas velocity basically controls the observed frequency, we call this the G regime.

\subsection{Convective/absolute transition}

In the previous two subsections, two types of spectra have been characterized as a function of the dynamic pressure ratio. In this section, we focus on the capability of the numerical computations and theoretical predictions to capture the 

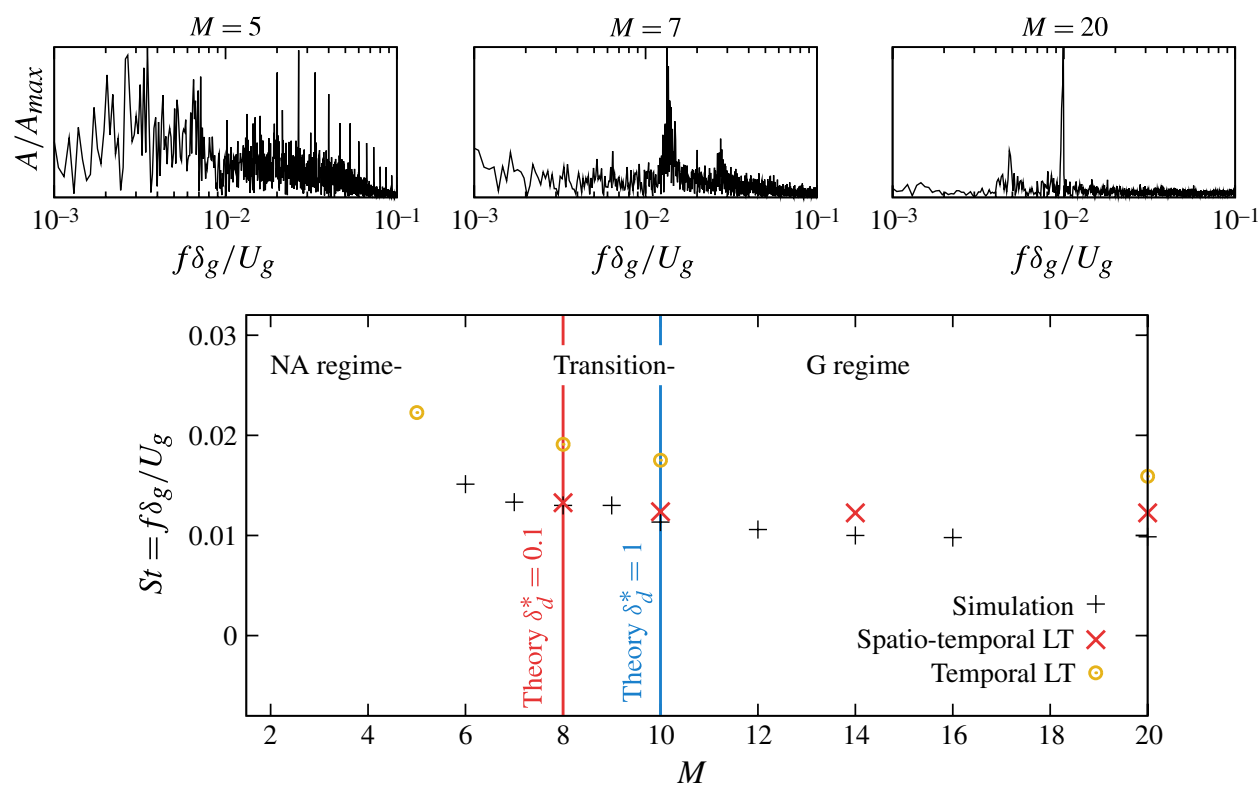

FIGURE 14. (Colour online) Amplitude of the wave and frequency of the most unstable frequency as a function of the dynamic pressure ratio for $r=0.1$ obtained from simulations $(+)$. As reference we include as vertical lines the prediction for the absolute/convective transition region from the linear theory results accounting for velocity defect $\left(M_{c}=8\right)$ and neglecting it $\left(M_{c}=10\right) . \times$, The frequency prediction of the spatio-temporal theory for $M=20$ accounting for the velocity defect; $\circ$, the prediction of the temporal linear theory. The FFT of the interface position at three different dynamic pressure ratios corresponding to each regime is included at the top of the figure (the frequency is given in non-dimensional units).

transition between these two regimes characterized by the nature of the instability (convective/absolute) (Huerre \& Monkewitz 1985, 1990). To this end, we perform a different set of simulations using different liquid velocities at constant gas velocity, and Reynolds and Weber numbers (table 1).

Figure 14 shows the most unstable frequency as a function of the dynamic pressure ratio. Two vertical lines have been used to delimit the transition predicted by the spatio-temporal linear theory between these two regimes, one accounting for the effect of the separator plate (the left-hand line) and the other neglecting it (the right-hand line). The spectra contained in figure 14 for different dynamic pressure ratios show the transition in the FFT from the appearance of the forcing frequencies at low dynamic pressure ratios to a clear peak at large ratios. In the $G$ regime, where the instability is absolute, we observe that the frequency is not significantly influenced either by the liquid velocity or by the dynamic pressure ratio. For low dynamic pressure ratios, the behaviour is more complex and one may have to consider all these parameters in order to understand how the most unstable frequency is influenced when the conditions are modified. For intermediate values, a transition regime appears where the peak is significantly broadened compared to the large dynamic pressure ratio limit.

When comparing the transition found numerically with the theoretical predictions, we clearly see that the presence of a velocity defect in the theoretical base velocity profile decreases the critical dynamic pressure ratio from 10 to 8 . As observed by 


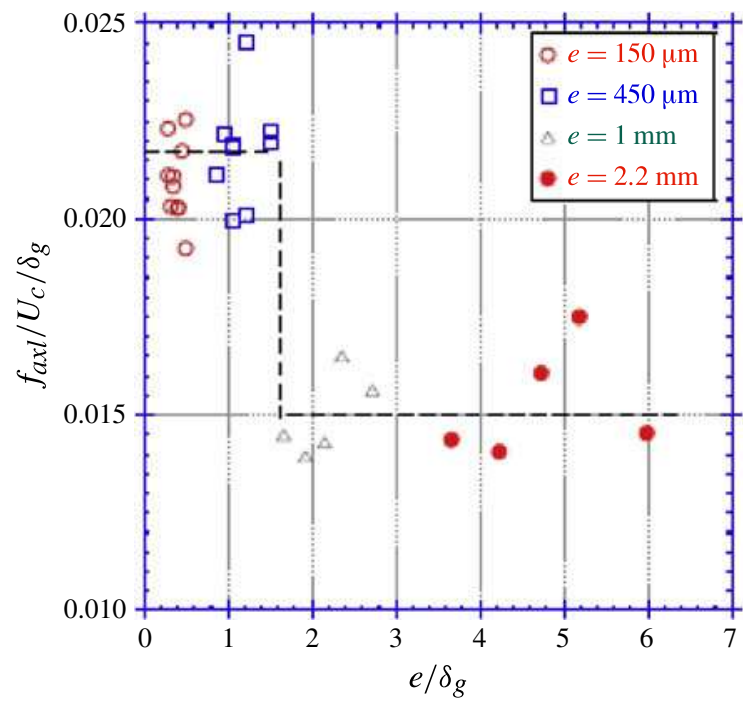

FIGURE 15. (Colour online) Experimental peak frequency as a function of the ratio $e / \delta_{g}$ at $M=16$. The measurements are taken at $x=5 \mathrm{~mm}$. Results extracted from Ben Rayana (2007). $U_{c}$ stands for the Dimotakis velocity $U_{D}$ (equation (5.3)).

Otto et al. (2013), the velocity defect induced by the presence of the injector tends to favour the appearance of an absolute instability. It is also important to note that the results from the spatio-temporal theory accounting for the velocity defect can be used to get a fairly good prediction of the critical dynamic pressure ratio for which absolute to convective transition occurs. The transition between the convective and absolute regime can be also seen, at constant gas velocity, as a critical liquid velocity ratio for which the flow structures are significantly different. This sudden change in the structure resembles the change already observed by Rehab, Villermaux \& Hopfinger (1997) for coaxial flows. In this work we will show in $\S 6.2$ that the features observed in figure 14 are qualitatively recovered in the planar flow configuration experimentally tested here.

\subsection{The I regime: the influence of the separator plate}

In the previous section we have considered scenarios where the thickness of the gas boundary layer is larger than that of the separator plate. However when the gas boundary layer becomes of the order of the thickness of the separator plate Ben Rayana (2007) has found that the splitter plate can have an impact on the most unstable frequency (figure 15).

In order to investigate this phenomenon, we perform three simulations in the simplified problem considered above with three different ratios $e / \delta_{g}$ (table 2).

Figure 16 depicts the FFT of the interface position behind the injector for $M=20$. As the ratio $e / \delta_{g}$ decreases, the peak observed is sharper. For Case $\mathrm{C}$, there is a sudden shift in the frequency for which we obtain the largest peak, although we still distinguish a peak in the observed frequency for those cases where the thickness of the gas boundary layer is larger than the thickness of the separator plate. Indeed, both frequencies are appreciable in all the simulations tested here. The largest peak appearing in Case $\mathrm{C}$ can be identified as a frequency peak induced by the presence of the injector, whereas the strongest peak in Cases A and B seems not to be 

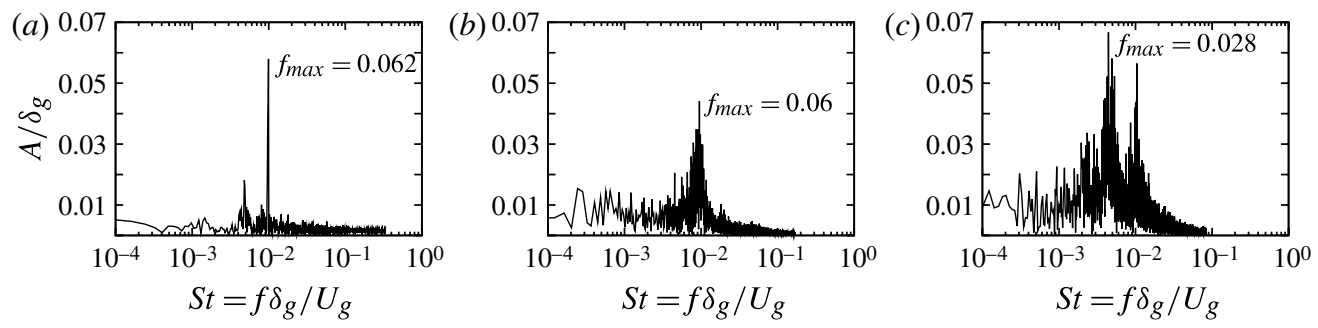

FIGURE 16. Simulation results. FFT of the position of the interface at $x / e=8$, for $M=20$, $r=0.1$, and $(a) e / \delta_{g}=0.5($ Case $A),(b) 1($ CaseB $),(c) 2($ Case $C)$. The forcing frequencies are not visible. The peak observed in the spectrum is more noisy for large $e / \delta_{g}$ ratios.

$\begin{array}{lcccccc}\text { Case } & e / \delta_{g} & \mu_{l} / \mu_{g} & W e_{g} & R e_{g} & r & M \\ \text { A } & 0.5 & 100 & 10 & 1000 & 0.1 & 20 \\ \text { B } & 1 & 100 & 10 & 1000 & 0.1 & 20 \\ \text { C } & 2 & 100 & 10 & 1000 & 0.1 & 20\end{array}$

TABLE 2. Simulation conditions for the analysis of the influence of the separator plate.

directly influenced by $e$, and can be attributed to the characteristics of the flow itself (velocities, properties, etc.). This observation is in agreement with the step function experimentally found by Ben Rayana (2007) included in figure 15, where the observed frequency is influenced by the presence of the separator plate for a splitter plate thickness larger than the threshold value given by $e / \delta_{g}=1$. As will be shown in $\S 6.3$, this effect is also found in the new measurements performed for air/water conditions, where the shift induced in the observed frequency due to the presence of the separator plate is of the order of two.

To sum up, the most unstable frequency is strongly determined by the flow behind the separator plate when the thickness of the gas boundary layer is of the order of the thickness of the separator plate. Thus, we name this region the injector-influenced regime or I regime. It is important to note that both frequencies may coexist in a transition region where both thicknesses are of the same order.

\subsection{Summary of results at low density ratios}

Figure 17 presents a phase diagram that summarizes the different regimes characterized in this section. For low dynamic pressure and small $e / \delta_{g}$ ratios the instability has been shown to be convective. In this regime (noise amplifier or NA regime), forcing frequencies grow or decay according to the linear theory predictions while the amplitudes of the disturbances are small enough. For large dynamic pressure ratios, there is a transition from convective to absolute instability. In this case, the $\mathrm{G}$ regime, a clear frequency appears in the spectrum irrespective of the forcing frequency which does not depend significantly on the liquid velocity. The spatiotemporal analysis provides a good estimate of the observed frequencies for most of the cases tested here. Linear theory results are also shown to provide a relatively accurate estimate of the critical dynamic pressure ratio for which regime transition occurs. For $e \ll \delta_{g}$, the separator plate has no influence on the base flow profile and the critical dynamic pressure ratio does not depend on the $e / \delta_{g}$ ratio. When the splitter 

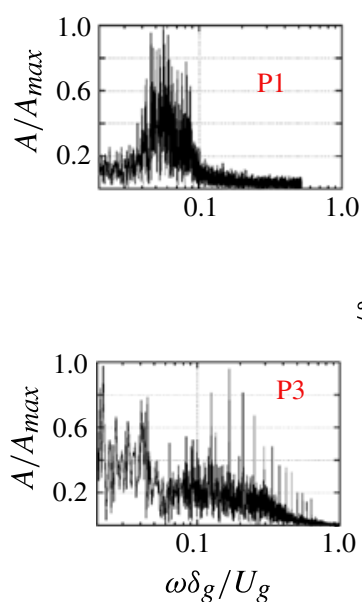

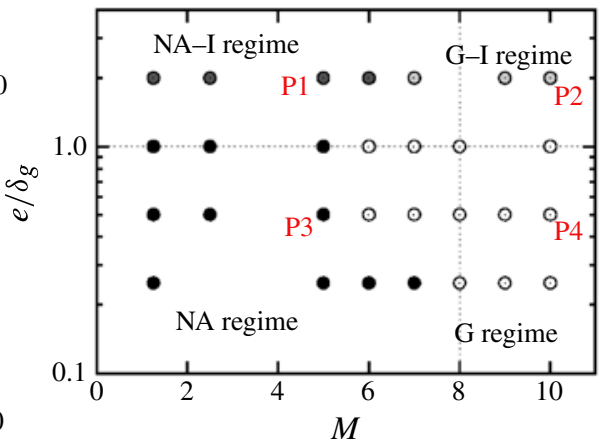

M
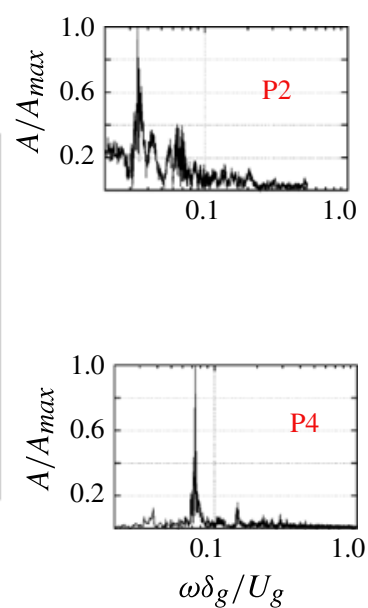

FIGURE 17. (Colour online) Regime map of the appearance of waves in the primary atomization region obtained from numerical simulation at $r=0.1$. The white circles represent a clear unique frequency appearing in the spectra at $x / e=8$, black circles indicate that the forcing frequencies can be clearly distinguished and grey circles indicate that two different frequency peaks of similar amplitude appear. From the results obtained we define the NA regime as the regime where small disturbance grow downstream and where we expect linear theory to apply, the I regime as the conditions for which the thickness of the separator plate has an impact on the observed frequencies and the $\mathrm{G}$ regime as where the global mode appears. For reference we include a horizontal line for $e / \delta_{g}=1$ and a vertical line at the critical dynamic pressure ratio for which the spatio-temporal linear analysis predicts transition from a convective to absolute instability. We also show characteristic FFT spectra of the interface height for each of the regimes characterized, labelled P1-P4.

plate thickness becomes of the order of the gas boundary layer, the critical dynamic pressure ratio is shifted to lower values. This fact reveals that the velocity defect tends to promote the appearance of a global instability compared to the limiting case where $e \ll \delta_{g}$.

Finally, when the $e / \delta_{g}$ ratio increases, a second peak appears in the spectrum due to the flow disturbances induced by the injector. This peak turns out to be the largest one, determining the observed frequency when $e / \delta_{g}>1$. We name this region the injector-influenced regime or I regime. The transition between the region where the separator plate controls the frequency is given by the ratio $e / \delta_{g} \approx 1$. For small $e / \delta_{g}$, the spatio-temporal theory provides a relatively good estimate of the critical value of $M$ for which the instability shifts from convective to absolute.

\section{Instability regime diagrams in air/water conditions}

In the previous section we have distinguished different instability regimes as a function of dynamic pressure ratio and the ratio between the gas boundary layer thickness and the splitter plate thickness. Using numerical results, the spatio-temporal linear theory has been shown to be a suitable tool to predict reasonably well the range of conditions for which we find a convective or an absolute instability. In this section, we extend the conclusions drawn in the previous section to air/water systems: numerical computations at large density ratios are compared to both new experimental data and to the spatio-temporal stability analysis. 


$\begin{array}{lcccc} & U\left(\mathrm{~m} \mathrm{~s}^{-1}\right) & \rho\left(\mathrm{kg} \mathrm{m}^{-3}\right) & \mu(\mathrm{Pa} \mathrm{s}) & \sigma\left(\mathrm{N} \mathrm{m}^{-1}\right) \\ \text { Gas } & 20 & 1.2 & 1.7 \times 10^{-5} & 0.069 \\ \text { Liquid } & 0.173 & 1000 & 10^{-3} & \end{array}$

TABLE 3. Physical properties of air and water at $20^{\circ} \mathrm{C}$ used as simulation conditions for the analysis of atomization processes.

$\begin{array}{ccccccc}m & r & R_{l} & R e_{g}^{*} & W e_{g}^{*} & M & \delta_{g} / \delta_{l} \\ \mu_{g} / \mu_{l} & \rho_{g} / \rho_{l} & \rho_{l} U_{l} H_{l} / \mu_{l} & \rho_{g} U_{g} \delta_{g} / \mu_{g} & \rho_{g}\left(U_{g}\right)^{2} \delta_{g} / \sigma & \frac{\rho_{g} U_{g}^{2}}{\rho_{l} U_{l}^{2}} & \\ 0.017 & 1.2 \times 10^{-3} & 1730 & 714 & 3.52 & 16 & 1\end{array}$

TABLE 4. Representative dimensionless numbers for the conditions given in table 3 , where $\mu$ is the viscosity, $\delta$ is the thickness of the boundary layer at the entrance, $\rho$ is the density and $\sigma$ is the surface tension.

We start by focusing on the measurements in the $G$ regime, the global instability regime, where a clear frequency peak appears in the spectrum. The peak in the spectra corresponds to the natural frequency present in the system, when no forcing is applied. In particular, for the comparison between numerical and experimental results we chose the conditions contained in table 3, which define the characteristic dimensionless parameters contained in table 4 .

\subsection{Numerical simulations of air/water systems in the G regime: comparison with theory and experiments}

\subsubsection{Problem set-up}

The numerical computation of the most unstable frequency is a challenging problem. The large density ratios encountered in air/water simulations significantly slow down the convergence of the Poisson solver. Also, we require large levels of refinement in the region close to the separator plate to correctly capture the physical mechanisms leading to the appearance of a physical instability. While we only consider a twodimensional case and use adaptive mesh refinement, the large domains required to minimize the influence of the boundaries on the measured frequencies and to describe a number of waves large enough to obtain representative statistics, lead to a very large number of cells. Thus, due to the high cost of the numerical computations, a detailed analysis of the different instability regimes described in the previous sections is not feasible with current computational resources and we restrict ourselves to the numerical analysis of a single case.

The simulation domain is a rectangular box where a standard outflow boundary condition (zero normal derivative of normal velocity and zero pressure) is imposed at the right-hand boundary of the domain whereas slip boundary conditions are set at the top and bottom boundaries as well as on the left-hand boundary in those zones where there is no entering jet. As stated above, if the output boundary condition is imposed before the jet is completely broken into droplets, some wave reflection can appear at the outflow boundaries significantly modifying the characteristic frequencies observed. In order to avoid this effect, a large domain of $16 \mathrm{~cm}$ is considered. The heights of 
(a)

$\Psi_{0}^{20}$

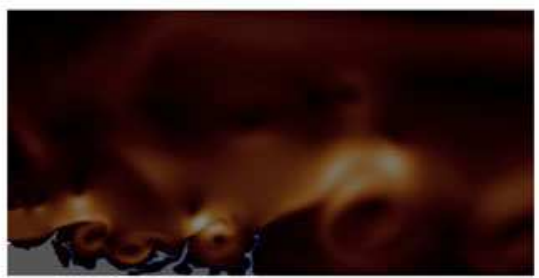

(b)

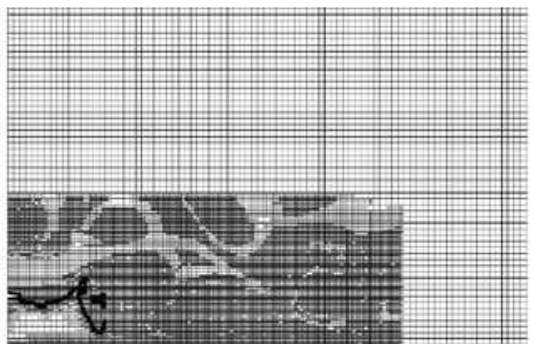

FIGURE 18. (Colour online) (a) Simulation domain and velocity norm field $\left(\mathrm{m} \mathrm{s}^{-1}\right)$. (b) Spatial mesh distribution (the view has been zoomed to appreciate the different grid levels).

(a)

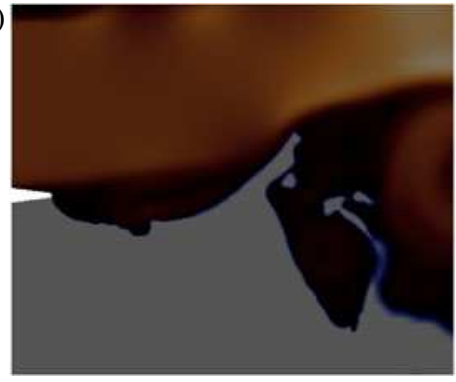

(b)

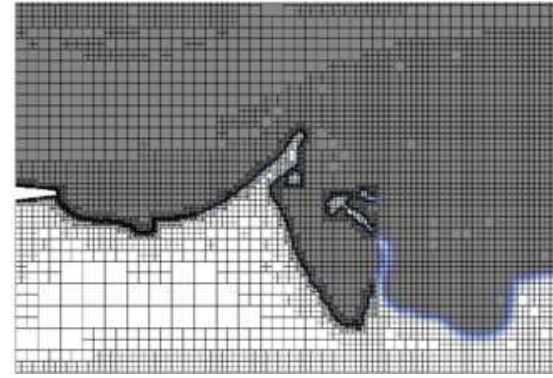

FIGURE 19. (Colour online) Zoomed view in the region near the splitter plate. (a) Velocity field (same colour scale as in figure 18). (b) Spatial mesh distribution.

the liquid and gas sheets at the entrance are, for both phases: $H_{g}=H_{l}=1 \mathrm{~cm}$. At the entrance, two boundary layers are introduced to reproduce the velocity profiles near the solid walls $\left(\delta_{l}=\delta_{g}=567 \mu \mathrm{m}\right)$. Between the liquid and gas jets, a separator plate with a minimum thickness of $150 \mu \mathrm{m}$ and a slope (or opening half-angle) of $\beta=3.5^{\circ}$ is included. Non-slip boundary conditions on the splitter plate walls or surface are imposed. The inclusion of the separator plate inside the simulation domain is important to capture some of the effects of the injector geometry on the flow (e.g. to capture the effect of the thickness of the separator plate $e$ and the opening half-angle $\beta$ ).

The mesh refinement capabilities have allowed us to consider a domain where the boundaries are relatively far from the injector without a prohibitive increase of the number of cells (see figure 18). For distances further than $2 \mathrm{~cm}$ from the injector, the mesh is progressively coarsened. Thus, although the accuracy of the results in this zone is not as good as near the injector (figure 19), the influence of the boundary conditions on the observed frequencies is negligible. In addition, very refined meshes can be used near the injector (of the order of $10 \mu \mathrm{m}$ ) which allows us to perform high-accuracy simulations of the flow in this zone. In particular, the maximum spatial resolution in the different parts of the domain is:

(i) $9.76 \mu \mathrm{m}$ for $x<2 \mathrm{~cm}$ and $y<3 \mathrm{~cm}$,

(ii) $39 \mu \mathrm{m}$ for $2<x<3 \mathrm{~cm}$ and $y<3 \mathrm{~cm}$,

(iii) $156 \mu \mathrm{m}$ for $x>3 \mathrm{~cm}$ and $y<3 \mathrm{~cm}$. 


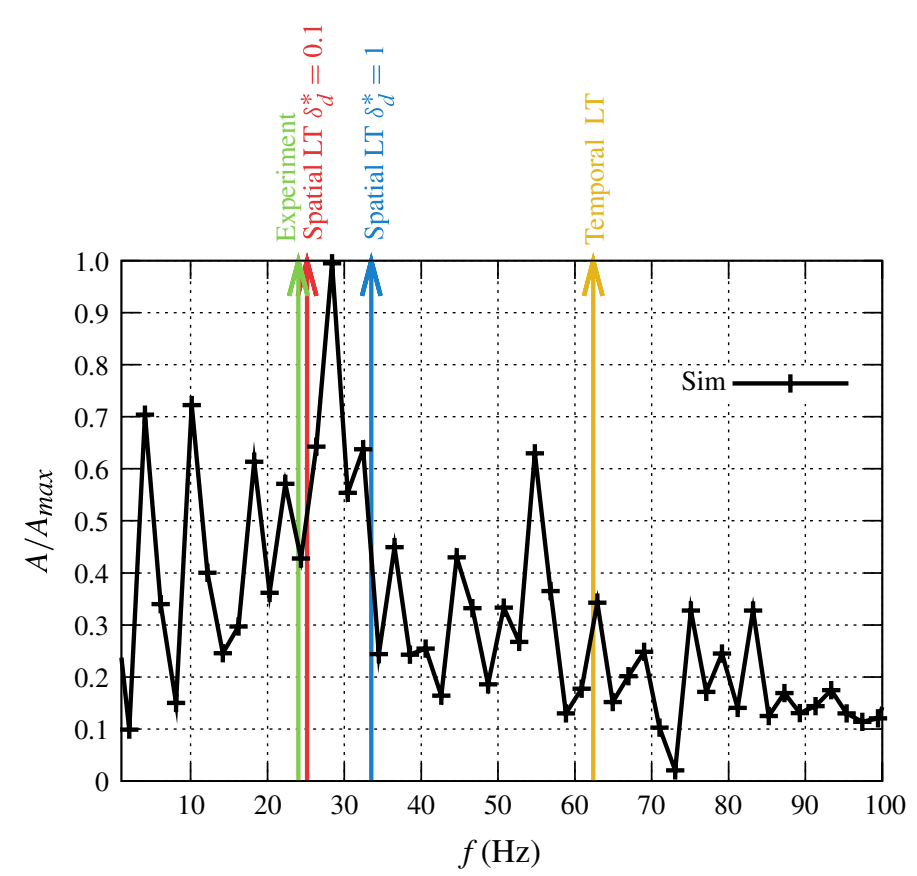

FIGURE 20. (Colour online) Fourier analysis of the interface height temporal evolution at $x / \delta_{g}=11$ obtained from numerical simulation, experiments and theoretical predictions by a temporal analysis and spatio-temporal analysis. Conditions of table 3. Arrows indicate the measured or predicted most unstable frequency, as labelled.

(iv) For $y>3 \mathrm{~cm}$, the interface is refined with cells of $156 \mu \mathrm{m}$ if some liquid reaches this zone. If no liquid is present, a coarse mesh is used to damp the vortices entering in this zone.

In addition, the grid near the solid walls is maximally refined $(9.76 \mu \mathrm{m})$ in order to maximize the accuracy of the simulation near the separator and to capture the generation of instabilities by the trailing edge of the splitter plate.

\subsubsection{Results}

The analysis of the simulation results reveals similar features to those observed in experiments and also in the moderate density ratio regime. Strong waves with a clear frequency appear from a region close to the splitter plate. The frequency obtained from simulation results matches relatively well the experimentally observed frequency for the same conditions (leftmost vertical arrow in figure 20). Note that unlike experimental results, numerical data are restricted to a narrow temporal window, which prevents obtaining a clear spectrum from numerical results. Nevertheless, the appearance of the resonant peak has been shown to become clearer as the simulation time is increased and to correspond relatively closely to the experimentally measured value.

For comparison, figure 20 also contains the temporal and spatio-temporal stability predictions of the most unstable frequencies. We observe a clear mismatch between the most unstable wavenumber experimentally observed and the most unstable one obtained from the linear stability analysis of the temporal problem, which is much 


$\begin{array}{lcccc}\begin{array}{l}\text { Raynal } \\ (1997)\end{array} & \begin{array}{c}\text { Marmottant \& } \\ \text { Villermaux } \\ (2004)\end{array} & \begin{array}{c}\text { Ben } \\ \text { Rayana } \\ (2007)\end{array} & \begin{array}{c}\text { Matas } \\ \text { et al. } \\ (2011)\end{array} & \begin{array}{c}\text { Present } \\ \text { study }\end{array} \\ 6 \times 10^{-4} & 7.5 \times 10^{-4} & 7.6 \times 10^{-4} & 5 \times 10^{-4} & 5 \times 10^{-4}\end{array}$

TABLE 5. Comparison of previously reported experimental values of $S t$ compared to the currently measured value.

larger. Remarkably, the theoretical results of the temporal linear theory are not significantly influenced by the base flow profile in this regime.

When considering the spatio-temporal problem, the appearance of a saddle point indicates the absolute nature of the instability. At the conditions tested in this section, the global nature of the instability appears irrespective of the velocity defect induced by the separator plate. At large $M=16$, the frequency only depends on gas velocity (as emphasized in figure 1). The limiting Strouhal number for the gas velocity tested here is found to be $S t_{\text {exp }}=f \delta_{g} / U_{g}=5 \times 10^{-4}$ experimentally and $S t_{\text {sim }}=8 \times 10^{-4}$ numerically. This value falls within the range of previously reported values as shown in table 5. The frequency match between simulation and experimental results indicates that the essential physical mechanisms leading to the appearance of characteristic frequencies in the interface oscillations are already captured with the current code for large density ratios.

\subsection{Absolute/convective transition}

The frequency is measured experimentally with the method described in $\S 2$, for a fixed gas velocity $\left(U_{g}=20 \mathrm{~m} \mathrm{~s}^{-1}\right)$, and for various liquid velocities, making the dynamic pressure ratio vary in the range [0.5-32]. Figure 21 shows that the experimental dimensionless frequency decreases as a function of $M$ (black dots). As mentioned in the previous subsection, the Strouhal value for large $M$ tends towards $5 \times 10^{-4}$, a value in agreement with previous works. For reference, we include the predictions from the linear theory, with and without a velocity defect (respectively crosses and plus signs). A good agreement is found for several values of $M$ when a velocity defect is included. Vertical lines indicate the limit between convective and absolute regimes predicted by the linear theory: the agreement in frequency corresponds to situations where the instability is absolute. This is consistent with the results of Otto et al. (2013) who find agreement between frequencies from linear theory and from experiment in the planar geometry only for situations corresponding to an absolute instability. When the instability is convective, predicted frequency is larger than experimental frequency, also as in Otto et al. (2013).

It has been shown in the previous section that in the case of moderate density ratios the existence of a narrow or broad maximum in the spectrum was sensitive to the dynamic pressure ratio. Figure 21 also presents spectra for three values of $M$ : the width of the spectrum decreases when $M$ is increased. This evolution is very similar to the one observed on figure 14: consistently with linear theory predictions, it suggests that the instability is convective for low $M$, and absolute for large $M$.

Note that the absolute regime observed when the velocity defect is accounted for appears irrespective of the far-field boundary conditions, which discards any influence of confinement on the appearance of a global instability as observed by Juniper \& Candel (2003), Juniper (2006) and Juniper et al. (2011). Note also that the critical 

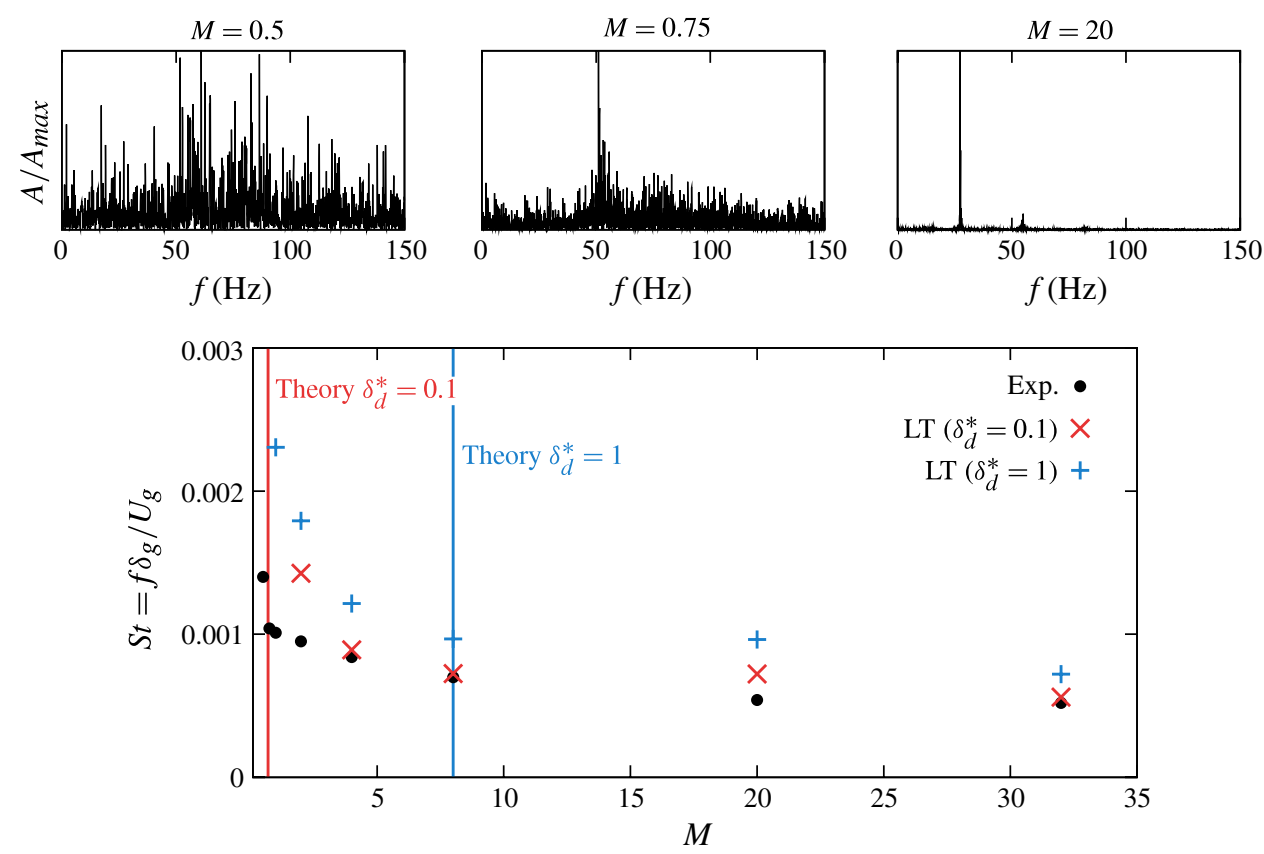

FIgURE 21. (Colour online) Experimental and theoretical results of the most unstable observed frequency as a function of the dynamic pressure ratio $(M)$ at constant gas velocity $\left(U_{g}=20 \mathrm{~m} \mathrm{~s}^{-1}\right)$. As reference we include linear theory (LT) results accounting for velocity defect $(x)$ and neglecting it $(+)$. The vertical lines represent the prediction for the absolute/convective transition region with and without velocity defect. The FFT of the interface position at three different dynamic pressure ratios corresponding to each regime are included at the top of the figure. The experimental regimes correspond well to the regimes identified from numerical simulations at moderate density ratios (figure 14).

dynamic pressure ratio that controls the absolute/convective transition is shifted to much lower values as the density ratio is increased: transition occurs at $M=8$ in figure 14 for $r=0.1$, while it occurs at around $M=1$ in figure 21 for $r=0.001$. One possible physical interpretation of this effect is that the amount of liquid swept along during the wave growth allows information to propagate upstream once we decrease the liquid velocity below a critical threshold. This makes the instability absolute. If we assume that the critical velocity evolves as the growth rate, $U_{l, c} \propto U_{g} \sqrt{r}$, the critical dynamic pressure ratio is expected to be

$$
M_{c}=r \frac{U_{g}^{2}}{U_{l, c}^{2}} \propto \sqrt{r} .
$$

This may explain quantitatively the shift observed in the critical dynamic pressure ratio between $r=0.1$ and $r=0.001$. This behaviour only appears when accounting for the velocity defect. If it is neglected the interpretation of the theoretical results in terms of absolute/convective transition is considerably more difficult. In this case, there are always branches appearing related to the finite domain size and it is difficult to conclude if the transition observed is due to confinement or due to the mechanism discussed above. 

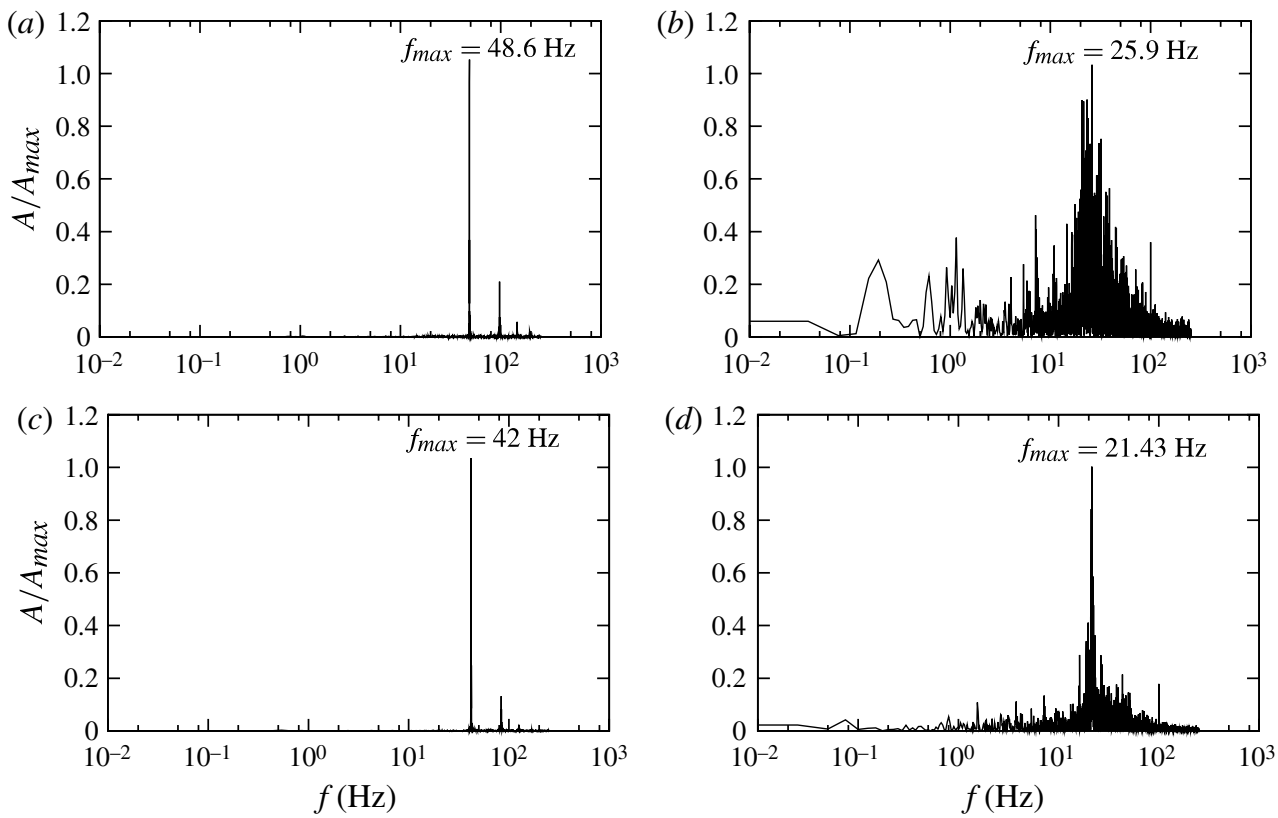

FIGURE 22. Experimental results in air/water conditions. FFT of the position of the interface for $e / \delta_{g}=0.38$ and 4.2 and dynamic pressure ratio 1.7 and 3.7 . $U_{g}=20 \mathrm{~m} \mathrm{~s}^{-1}$. When $e<\delta_{g}$ a clear peak appears in the spectrum. The spectrum becomes broad and noisy for $e>\delta_{g}$. (a) $M=1.7, e / \delta_{g}=0.38 ;(b) M=1.7, e / \delta_{g}=4.2 ;$ (c) $M=3.7, e / \delta_{g}=0.38 ;(d)$ $M=3.7, e / \delta_{g}=4.2$.

\subsection{The I regime: the influence of the separator plate}

The simulations at moderate density ratios have revealed the importance of the splitter plate in situations where the thickness becomes of the order of the vorticity layer thickness. In this section, we conduct new experiments in air/water conditions with two separator plates of thickness $e=0.2 \mathrm{~mm}$ and $e=2.2 \mathrm{~mm}$. The gas velocity is set to $U_{g}=20 \mathrm{~m} \mathrm{~s}^{-1}$ and the liquid velocity is adjusted to obtain the required dynamic pressure ratio. We test two different dynamic pressure ratios above the critical dynamic pressure ratio: $M=1.7$ and $M=3.7$. As in the simulation results, the spectra obtained when the thickness of the separator plate is larger than the gas boundary layer thickness is significantly noisier than when $e<\delta_{g}$. Furthermore, the observed frequencies are shifted to lower values (figure 22). For the test considered here, the frequency is shifted by a factor almost 2. Although it has not been possible to reproduce the influence of the splitter plate in air/water conditions in the simulation, the good qualitative agreement between the experimental results and the numerical simulations at moderate density ratios is remarkable (compare spectra of figure 16 and of figure 22). The fact that both numerical and experimental spectra become more noisy when $e / \delta_{g}$ increases is analogous to the transition from absolute to convective instability observed when the dynamic pressure ratio is decreased (see figures 14 and 21). This is counterintuitive because thick splitter plates help to create large velocity deficits, and therefore one would instead expect them to enhance absolute instability. Simulation results have revealed that the contact line travels up and down the leading edge of the separator plate, which may explain the significant amount of noise observed in the spectra. However it is difficult to conclude whether the 
dynamics of the contact line are responsible for the frequency shift or not. Because the model for contact line dynamics in simulation results is very crude and inaccurate (the interface moves using the standard VOF method), it does not seem reasonable to assume that a process controlled by contact line dynamics would be accurately captured. We therefore believe that the frequency shift is a consequence of the flow created behind the separator plate and not the contact line dynamics. In any case, this phenomenon certainly requires further investigations in the future.

\section{Conclusions}

This work investigates the mechanism of wave generation in primary atomization. The numerical simulations show the capability of the code to correctly capture the main mechanisms leading to the appearance of clear frequencies observed in air/water conditions, where the frequency peak obtained from the simulation fits well both theoretical and experimental results.

We have identified three different flow regimes when the dynamic pressure ratio and the ratio $e / \delta_{g}$ are varied. When the gas boundary layer is larger than the separator plate thickness a transition from convective to absolute instability occurs when increasing the dynamic pressure ratio at constant gas velocity. This transition has been numerically investigated for small density ratios and experimentally validated at air/water conditions. In both cases, the spatio-temporal theory proposed by Otto et al. (2013) with a base flow accounting for the velocity defect (as in Matas et al. 2011) predicts relatively well the transition from convective to absolute instability when the dynamic pressure ratio increases. For small dynamic pressure ratios a band of frequencies are amplified in the simulation tests included here. This behaviour is consistent with the convective nature of the instability predicted by the spatio-temporal linear theory which also reproduces reasonably well the range of frequencies amplified in numerical simulations. For large dynamic pressure ratios, a strong instability appears just behind the separator plate and a clear frequency peak is observed irrespective of the downstream position. This type of behaviour is related to the appearance of an absolute instability, which is predicted by the spatio-temporal linear theory. In addition, in agreement with previous experiments reported in the literature (Raynal 1997; Marmottant \& Villermaux 2004; Ben Rayana 2007; Matas et al. 2011) and theoretical predictions, numerical computations correctly capture the frequency scaling with the gas velocity. In this regime, the observed frequency is not significantly influenced either by the liquid boundary layer or by the liquid velocity. A third regime is observed for large splitter plates: the presence of the separator plate has been shown to have a significant impact on the observed frequencies given that it modifies the base flow profile. Numerical and experimental results have also shown than when the splitter plate thickness is of the order of the gas boundary layer thickness the interference of the splitter plate tends to reduce the observed frequency, make the resonant peak wider and introduce a significant noise in the spectrum.

As stated in the introduction, it is widely accepted that the instability of a two-phase mixing layer is controlled by the gas vorticity thickness $\delta_{g}$ : inviscid models accounting for that vorticity thickness and also for a velocity deficit in the initial profiles (Matas et al. 2011) succeed in capturing the correct scaling of the frequency of the unstable mode as a function of $U_{g}$, in particular at large $M$ (see figure 1) but they fail to reproduce experimental growth rates. We show in the present paper that in addition to better capturing experimental growth rates (Otto et al. 2013), a spatio-temporal viscous linear stability analysis performed on the same base flow profile also captures the 
same scaling for the frequency (figure 13). Moreover, we also show that experimental frequencies are correctly reproduced for large $M$, when the instability is predicted to be absolute (figure 21): the physical mechanism at play, and the question of why simple inviscid models also perform relatively well remain open issues. In any event, current theoretical, experimental and simulation results point out that the velocity defect induced by the separator plate is responsible for the appearance of an absolute instability. At small $M$, the situation is even more delicate as the spatio-temporal analysis has problems in predicting experimentally observed frequencies. The issue here may be related to the high sensitivity of the spatio-temporal analysis to the details of the base velocity profile.

More generally it is worth emphasizing that the predictions of spatio-temporal analyses strongly depend on the inclusion of a velocity defect, in agreement with the strong influence of the splitter plate on experimental and numerical results. In real situations the base flow close to the interface is expected to undergo strong spatial variations: a better understanding of the role of the splitter plate is therefore crucial to better predict wave growth and frequency, which in turn control drop size and fluxes in applications related to atomization.

\section{Acknowledgements}

The authors would like to acknowledge Dr T. Otto and Dr T. Boeck for sharing their code and their useful comments and suggestions interpreting the results obtained from the spatio-temporal stability code. This project has been supported by the ANR VAA (ANR-2010-BLAN-0903) and ANR DYNAA (ANR-2005-BLAN-0213) programs, the ANR MODEMI ANR-2011-MONU-0011 and the FIRST project supported by the European Commission under the 7th Framework Programme.

\section{REFERENCES}

Agbaglah, G., Delaux, S., Hoepffner, D., Fuster, J., Josserand, C., Popinet, S., Ray, P., Scardovelli, R. \& ZAleski, S. 2011 Parallel simulation of multiphase flows using octree adaptivity and the volume-of-fluid method. C. R. Acad. Sci. Paris 339, 194-207.

Bagué, A., Fuster, D., Popinet, S., Scardovelli, R. \& Zaleski, S. 2010 Instability growth rate of two-phase mixing layers from a linear eigenvalue problem and an initial value problem. Phys. Fluids 22 (9), 092104.

BEN RAYANA, F. 2007 Contribution à l'étude des instabilités interfaciales liquide-gaz en atomisation assistée et taille de gouttes. PhD thesis, Institut National Polytechnique de Grenoble.

Ben Rayana, F., Cartellier, A. \& Hopfinger, E. 2006 Assisted atomization of a liquid layer: investigation of the parameters affecting the mean drop size prediction. In Proceedings ICLASS 2006, August 27-September 1, Kyoto Japan. Academic, iSBN4-9902774-1-4.

Bianchi, G. M., Pelloni, P., Toninel, S., Scardovelli, R., Leboissetier, A. \& Zaleski, S. 2005 A quasi-direct 3D simulation of the atomization of high-speed liquid jets. In Proceedings of ICESO5, 2005 ASME ICE Division Spring Technical Conference. Chicago, Illinois, USA, April 5-7, 2005.

BOECK, T. \& ZALESKI, S. 2005 Viscous versus inviscid instability of two-phase mixing layers with continuous velocity profile. Phys. Fluids 17, 032106.

Dimotakis, P. E. 1986 Two-dimensional shear-layer entrainment. AIAA J. 24, 1791-1796.

Eggers, J. \& Villermaux, E. 2008 Physics of liquid jets. Rep. Prog. Phys. 71, 036601.

Fuster, D., Agbaglah, G., Josserand, C., Popinet, S. \& Zaleski, S. 2009a Numerical simulation of droplets, bubbles and waves: state of the art. Fluid Dyn. Res. 41, 065001.

Fuster, D., Bagué, A., Boeck, T., Le Moyne, L., Leboissetier, A., Popinet, S., Ray, P., SCARdovelli, R. \& ZALESKI, S. $2009 b$ Simulation of primary atomization with an octree adaptive mesh refinement and VOF method. Intl J. Multiphase Flow 35, 550-565. 
Gordillo, J. M., Perez-Saborid, M. \& Gañan-Calvo, A. M. 2001 Linear stability of co-flowing liquid-gas jets. J. Fluid Mech. 448, 23-51.

Gorokhovski, M. \& Herrmann, M. 2008 Modelling primary atomization. Annu. Rev. Fluid Mech. 40, 343-366.

Huerre, P. \& Monkewitz, P. 1985 Absolute and convective instabilities in free shear layers. J. Fluid Mech. 159, 151-168.

Huerre, P. \& Monkewitz, P. A. 1990 Local and global instabilities in spatially developing flows. Annu. Rev. Fluid Mech. 22, 473-537.

JUNIPER, M. P. 2006 The effect of confinement on the stability of two-dimensional shear flows. J. Fluid Mech. 565, 171-195.

Juniper, M. P. \& CANDEL, S. M. 2003 The stability of ducted compound flows and consequences for the geometry of coaxial injectors. J. Fluid Mech. 482, 257-269.

Juniper, M. P., TAmmisola, O. \& Lundell, F. 2011 The local and global stability of confined planar wakes at intermediate Reynolds number. J. Fluid Mech. 686, 218-238.

Lasheras, J. C. \& Hopfinger, E. J. 2000 Liquid jet instability and atomization in a coaxial gas stream. Annu. Rev. Fluid Mech. 32, 275-308.

Lasheras, J. C., Villermaux, E. \& Hopfinger, E. J. 1998 Break-up and atomization of a round water jet by a high-speed annular air jet. J. Fluid Mech. 357, 351-379.

Marmottant, P. \& VillermauX, E. 2004 On spray formation. J. Fluid Mech. 498, 73-111.

Matas, J. P., MARTy, S. \& CARTellier, A. 2011 Experimental and analytical study of the shear instability of a gas-liquid mixing layer. Phys. Fluids 23, 094112.

Menard, T., Tanguy, S. \& Berlemont, A. 2007 Coupling level set/VOF/ghost fluid methods: validation and application to 3D simulation of the primary break-up of a liquid jet. Intl $J$. Multiphase Flow 33, 510-524.

Ó’NÁraigh, L., Spelt, P. D. M. \& Shaw, S. J. 2013 Absolute linear instability in laminar and turbulent gas-liquid two-layer channel flow. J. Fluid Mech. 714, 58-94.

Ó’NÁRAigh, L., SPELT, P. D. M. \& ZAKI, T. A. 2011 Turbulent flow over a liquid layer revisited: multi-equation turbulence modelling. J. Fluid Mech. 683, 357-394.

Отто, T., Rossi, M. \& BOECK, T. 2013 Viscous instability of a sheared liquid-gas interface: dependence on fluid properties and basic velocity profile. Phys. Fluids 25, 032103.

Popinet, S. 2003 Gerris: a tree-based adaptive solver for the incompressible Euler equations in complex geometries. J. Comput. Phys. 190 (2), 572-600.

Popinet, S. 2009 An accurate adaptive solver for surface-tension-driven interfacial flows. J. Comput. Phys. 288, 5838-5866.

RaYleigh, Lord \& Baron, J. W. S. 1964 Scientific Papers. Dover.

RAYNAL, L. 1997 Instabilité et entraînement à l'interface d'une couche de mélange liquide-gaz. PhD thesis, Université Joseph Fourier, Grenoble.

REeS, S. J. \& JUNIPER, M. P. 2010 The effect of confinement on the stability of viscous planar jets and wakes. J. Fluid Mech. 656, 309-336.

Rehab, H., Villermaux, E. \& Hopfinger, E. J. 1997 Flow regimes of large-velocity-ratio coaxial jets. J. Fluid Mech. 345 (1), 357-381.

Sahu, K. C., Valluri, P., Spelt, P. D. M. \& Matar, O. K. 2007 Linear instability of pressure-driven channel flow of a Newtonian and a Herschel-Bulkley fluid. Phys. Fluids 19, 122101.

Shinjo, J. \& UMemura, A. 2010 Simulation of liquid jet primary breakup: dynamics of ligament and droplet formation. Intl J. Multiphase Flow 36, 513-532.

Shinjo, J. \& UMEMURA, A. 2011 Surface instability and primary atomization characteristics of straight liquid jet sprays. Intl J. Multiphase Flow 37, 1294-1304.

Tomar, G., Fuster, D., Zaleski, S. \& Popinet, S. 2010 Multiscale simulations of primary atomization using gerris. Comput. Fluids 39 (4), 1864-1874.

Yecko, P. \& ZALESKI, S. 2005 Transient growth in two-phase mixing layers. J. Fluid Mech. 528, $43-52$.

Yecko, P., Zaleski, S. \& Fullana, J.-M. 2002 Viscous modes in two-phase mixing layers. Phys. Fluids 14, 4115-4122. 